\title{
QCD resummation on single hadron transverse momentum distribution with the thrust axis
}

\author{
Zhong-Bo Kang, ${ }^{a, b, c}$ Ding Yu Shao ${ }^{a, b, c}$ and Fanyi Zhao ${ }^{a, b}$ \\ ${ }^{a}$ Department of Physics and Astronomy, University of California, \\ Los Angeles, CA 90095, U.S.A. \\ ${ }^{b}$ Mani L. Bhaumik Institute for Theoretical Physics, University of California, \\ Los Angeles, CA 90095, U.S.A. \\ ${ }^{c}$ Center for Frontiers in Nuclear Science, Stony Brook University, \\ Stony Brook, NY 11794, U.S.A. \\ E-mail: zkang@physics.ucla.edu, dingyu.shao@physics.ucla.edu, \\ fanyizhao@physics.ucla.edu
}

ABSTRACT: We derive the transverse momentum dependent (TMD) factorization and resummation formula of the unpolarized transverse momentum distribution $\left(j_{T}\right)$ for the single hadron production with the thrust axis in an electron-positron collision. Two different kinematic regions are considered, including small transverse momentum limit $j_{T} \ll Q$, and joint transverse momentum and threshold limit $j_{T} \ll Q\left(1-z_{h}\right) \ll Q$, where $Q$ and $z_{h}$ are the hard scattering energy and the observed hadron momentum fraction. Using effective theory methods, we resum logarithms $\ln \left(Q / j_{T}\right)$ and $\ln \left(1-z_{h}\right)$ to all orders. In the end, we present the differential cross sections and Gaussian widths calculated for the inclusive charged pion production and find that our results are consistent with the measurements reported by the Belle collaboration.

KeYwords: Perturbative QCD, Resummation, Effective Field Theories

ArXiv EPrint: 2007.14425 


\section{Contents}

1 Introduction 1

2 TMD formalism: global structure 3

2.1 TMD factorization formalism 4

$\begin{array}{lr}2.2 & \text { TMD formalism in coordinate space }\end{array}$

$\begin{array}{ll}2.3 \text { TMD formalism at threshold } z_{h} \rightarrow 1 & 10\end{array}$

3 Factorization and resummation: full story 14

$\begin{array}{llr}4 & \text { Numerical results } & 16\end{array}$

5 Conclusion $\quad 20$

\section{Introduction}

The transverse momentum dependent parton distribution functions (TMD PDFs) and fragmentation functions (TMD FFs) are the fundamental objects to understand the intrinsic hadron structure, in particular, the three-dimensional (3D) imaging of the hadrons in the momentum space [1-3]. A lot of progress has been made in understanding 3D imagining of the nucleon via both unpolarized and polarized TMD PDFs, see some recent work in refs. [4-15]. On the other hand, in comparison with the fruitful results in TMD PDFs, the research on the TMD FFs of hadrons definitely needs more development. The current main channels to probe TMD FFs are either semi-inclusive processes in deep inelastic scattering (SIDIS) and $e^{+} e^{-}$collisions (hadron pair production) [16-19], or hadron distribution inside jets [20-26]. For a recent review on fragmentation functions, see ref. [27]. It will be interesting and instructive to find more observable to probe TMD FFs.

The extraction of TMD PDFs and/or TMD FFs relies on the so-called TMD factorization in QCD. For example, transverse momentum distribution of the Drell-Yan type processes has been developed in the seminal literature by Collins, Soper, and Sterman [28] for a long time and is usually referred to as CSS formalism. For a modern reformulation of the CSS formalism, see [29]. Similar formulas describe the TMD factorization for the SIDIS process in electron-nucleon collisions, $e^{-} p \rightarrow e^{-} h X$ [16, 30-32], and for back-to-back hadron pair production in $e^{+} e^{-}$annihilation, $e^{+} e^{-} \rightarrow h_{1} h_{2} X$ [33-35]. The universality for the non-perturbative parametrization has been investigated in $[5,6,17,36-40]$. Recently, the TMD factorization structure has been re-investigated using Soft-Collinear Effective Theory (SCET) [41-44] and the renormalization group (RG) techniques [45-47].

The electron-positron collider provides a clean environment to study TMD FFs using the inclusive hadron production since there is no hadronic contamination from the initial 
states. The standard process to probe TMD FFs in $e^{+} e^{-}$collisions is the aforementioned back-to-back hadron pair production, $e^{+} e^{-} \rightarrow h_{1} h_{2} X$, which probes the same TMD FFs as those in SIDIS process, $e^{-} p \rightarrow e^{-} h X .{ }^{1}$ Recently, the single-hadron differential cross section for the process, $e^{+} e^{-} \rightarrow h X$, is reported by the Belle collaboration [48], where the hadron cross section is studied as a function of the event-shape variable called thrust $T$, fractional energy $z_{h}$, and the transverse momentum $j_{T}$ with respect to the thrust axis. The $j_{T}$ distribution shows the usual Gaussian shape and this gives the hope that through such a new measurement, one would gain a better understanding of the same TMD FFs. With such an assumption, some phenomenological work has been performed in [49, 50].

In this paper, we perform a detailed theoretical study for the Belle observable and develop a TMD factorization formalism for describing such a $j_{T}$ distribution. The plane perpendicular to the thrust axis splits the full phase space into two hemispheres. One only measures the hadron $j_{T}$ in one hemisphere, and the other hemisphere is unmeasured. Such type of measurements is termed as non-global observables [51], which are sensitive to radiation in only a part of phase space. The factorization and resummation formula for nonglobal observables have a very different structure from the standard global observable [52]. For example, the leading-order evolution equation for non-global logarithms (NGLs), the BMS equation [53], is a non-linear evolution equation. The TMD factorization formalism for the non-global hemisphere event shape has been studied by one of the authors in [54], where they find that the rapidity logarithms evolution does not constitute an essential complicated structure, since it is tied with a universal transverse momentum dependent jet function which also appears in the global observables. Besides, after comparing with the data at the LEP, they also find that the leading non-perturbative effects are related to the Collins-Soper kernel. ${ }^{2}$

We mainly consider the kinematic region with $j_{T} \ll Q$, where a TMD factorization can be developed which resums $\ln \left(Q / j_{T}\right)$. Here $Q$ is the virtuality of the intermediate photon in $e^{+} e^{-} \rightarrow \gamma^{*}$. In this region, Belle collaboration finds that the cross sections can be well described by Gaussians in $j_{T}$, and that the width of the Gaussians shows an initially rising, then decreasing $z_{h}$-dependence when $z_{h} \rightarrow 1$. Because of this, we further consider the threshold $\ln \left(1-z_{h}\right)$ resummation in the $z_{h} \rightarrow 1$ limit. We apply SCET to develop a TMD factorization formalism. Using renormalization group evolution techniques, we resum logarithmic terms to next-to-leading logarithmic (NLL) accuracy, including NGLs. The experimental data are shown as comparison and in good agreement with our theoretical predictions.

The remainder of this paper is organized as follows. In section 2, we present a factorized framework, which only resums the so-called global logarithms. This section would allow us to develop intuition for our framework and understand connection to the standard TMD FFs. In section 3, we present the full factorization formalism, which allows us to resum both global and NGLs. In section 4, numerical results of differential cross sections for

\footnotetext{
${ }^{1}$ Note that the modern formulation of so-called properly-defined TMD FFs combines the usual TMD FFs and the soft function for the process. Here we are referring to the properly-defined TMD FFs. For details, see [29].

${ }^{2}$ In [54] the Collins-Soper kernel is named as the collinear anomaly function.
} 
pion production in $e^{+} e^{-} \rightarrow \pi^{ \pm} X$ are presented, as a function of energy fraction $z_{h}$ and transverse momentum $j_{T}$. We also present the Gaussian width for the $j_{T}$ distribution as computed from our theoretical formalism, and compare them with the Belle experimental data. Finally, conclusions are given in section 5 .

\section{TMD formalism: global structure}

We consider the process, $e^{+}+e^{-} \rightarrow h\left(z_{h}, j_{T}\right)+X$, in $e^{+} e^{-}$annihilation. The center-ofmass $(\mathrm{CM})$ energy of the $e^{+} e^{-}$collisions is given by $s=Q^{2}=\left(p_{e^{+}}+p_{e^{-}}\right)^{2}$, and the hadron momentum fraction $z_{h}=2 p_{h} \cdot q / Q^{2}=2 E_{h} / Q$ is measured. In addition, the hadron's transverse momentum $j_{T}$ is measured with respect to the so-called thrust axis $\hat{n}$, which maximizes the event-shape variable thrust $T$ [55]:

$$
T \equiv \max _{\hat{n}} \frac{\sum_{i}\left|\vec{p}_{i} \cdot \hat{n}\right|}{\sum_{i}\left|\vec{p}_{i}\right|}
$$

with the momenta $\vec{p}_{i}$ of the particles measured in the $e^{+} e^{-}$CM frame. For convenience, we align the thrust axis to be along $+z$-direction, and define light-like vectors $n^{\mu}=(1, \hat{n})=$ $(1,0,0,1)$ and $\bar{n}^{\mu}=(1,-\hat{n})=(1,0,0,-1)$. We expand any momentum $p^{\mu}$ in the light cone frame as $p^{\mu}=\left(p^{+}, p^{-}, p_{T}\right)$ with $p^{+}=n \cdot p=p_{0}-p_{z}$ and $p^{-}=\bar{n} \cdot p=p_{0}+p_{z}$. It is important to emphasize that even though we measure the hadron transverse momentum $j_{T}$ with respect to the thrust axis, our cross section is not differential in the thrust variable $T$. In other words, we consider the cross section for the hadron production, which is differential only in $z_{h}$ and $j_{T}$ :

$$
\frac{d \sigma}{d z_{h} d^{2} \vec{j}_{T}}
$$

That is to say, the only purpose of the thrust measurement is to provide the thrust axis $\hat{n}$ and we sum over the entire thrust region $0.5<T<1$. For the cross section that is further differential in the thrust variable $T$, see e.g. refs. [56]. We find that such an observable in eq. (2.2) has a better connection to the standard TMD FFs.

The plane perpendicular to the thrust axis divides the full space into two hemispheres: the one on the right (along $+z$-side) is referred to as the right hemisphere, while the one on the left is the left hemisphere. Note that the observed hadrons are always measured in the right hemisphere, while no measurement is performed for the left hemisphere. Because of this, the kinematics in the left hemisphere are unconstrained, our observable in eq. (2.2) is a non-global observable [57]. Such observables will involve non-global structures which can not be captured by the traditional exponential formula [52]. In this paper we will apply the jet effective theory $[58,59]$ to derive the factorization and resummation formula. Since the full factorization structure is quite complicated which we save for the next section, in this section, we will for the moment ignore the NGLs that arise from the non-global structure, and write down a factorized formalism to resum the global logarithms and build our intuition. 

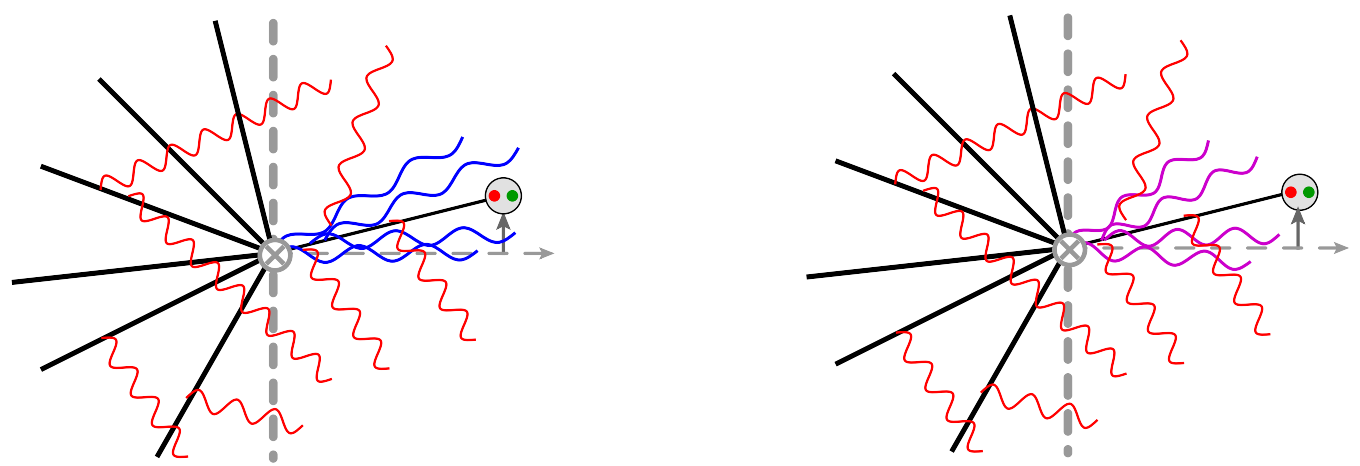

Figure 1. Hadron transverse momentum $\vec{j}_{T}$ with the thrust axis $\hat{n}$ in two different regions. The black lines represent the energetic partons in the unmeasured left hemisphere, while the hadron is measured in the right hemisphere. The vertical dashed line represents a plane that is perpendicular to the thrust axis and that divides the space to the left and right hemisphere. The red curves denote soft radiations from the energetic partons with the virtuality of $j_{T}$. The blue lines in the left panel describe collinear radiations along the thrust axis, while the purple ones in the right panel give collinear-soft (c-soft) radiations.

\subsection{TMD factorization formalism}

We consider the kinematic region where the transverse momentum is small $j_{T} \ll Q$ and thus is sensitive to TMD physics. Setting the usual power expansion parameter $\lambda=j_{T} / Q$, we find that the relevant momentum modes in this region are given by

- hard: $\quad p_{h} \sim Q(1,1,1)$

- collinear: $p_{c} \sim Q\left(\lambda^{2}, 1, \lambda\right)$

- soft: $\quad p_{s} \sim Q(\lambda, \lambda, \lambda)$

The different modes are illustrated in figure 1 (left). The hard modes encode energetic radiations in the left hemisphere: since the hadron is observed in the right hemisphere and has $j_{T} \ll Q$, any energetic radiation in the right hemisphere will lead to a large transverse momentum for the hadron and thus move the hadron out of the kinematic $j_{T} \ll Q$ region; consequently such radiation is not allowed in the right hemisphere. On the other hand, soft and collinear modes have the same transverse momentum of $j_{T}$, and thus both contribute to our observable. The difference is that collinear modes encode energetic radiations along the thrust axis, while soft modes describe large-angle long wave radiations. Based on the mode analysis, the factorization formalism is given as

$$
\begin{aligned}
\frac{d \sigma}{d z_{h} d^{2} \vec{j}_{T}}= & \sigma_{0} \sum_{i=q, \bar{q}, g} e_{q}^{2} \int d^{2} \vec{k}_{T} d^{2} \vec{\lambda}_{T} \delta^{(2)}\left(\vec{j}_{T}-\vec{k}_{T}-z_{h} \vec{\lambda}_{T}\right) \\
& \times \mathcal{H}^{i}(Q, \mu) D_{h / i}\left(z_{h}, k_{T}, \mu, \nu\right) \mathcal{S}_{i}\left(\lambda_{T}, \mu, \nu\right)
\end{aligned}
$$




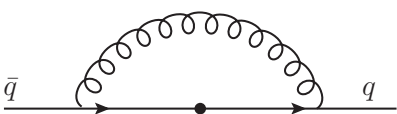

(a)

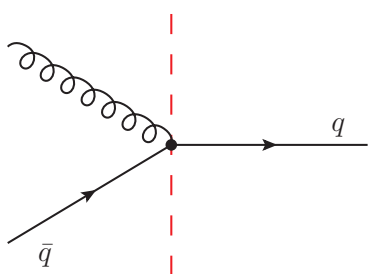

(b)

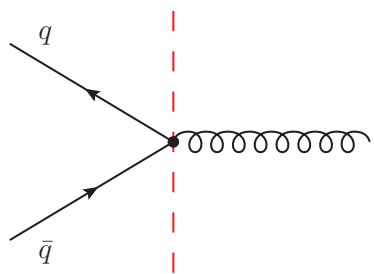

$(c)$

Figure 2. Three configurations that contribute to the NLO hard function: (a) virtual correction; (b) quark $q$ is on the right hemisphere, while both anti-quark $\bar{q}$ and gluon $g$ are on the left hemisphere; (c) gluon $g$ is on the right hemisphere, while both quark $q$ and anti-quark $\bar{q}$ are on the left hemisphere. Note that the observed hadron is on the right hemisphere.

where the summation $\sum_{i=q, \bar{q}, g} e_{q}^{2} \cdots$ on the right hand side is interpreted as

$$
\begin{aligned}
\sum_{q=u, d, s, \cdots} & e_{q}^{2} \int d^{2} \vec{k}_{T} d^{2} \vec{\lambda}_{T} \delta^{(2)}\left(\vec{j}_{T}-\vec{k}_{T}-z_{h} \vec{\lambda}_{T}\right) \\
\times & {\left[\mathcal{H}^{q}(Q, \mu) D_{h / q}\left(z_{h}, k_{T}, \mu, \nu\right) \mathcal{S}_{q}\left(\lambda_{T}, \mu, \nu\right)+\mathcal{H}^{\bar{q}}(Q, \mu) D_{h / \bar{q}}\left(z_{h}, k_{T}, \mu, \nu\right) \mathcal{S}_{\bar{q}}\left(\lambda_{T}, \mu, \nu\right)\right.} \\
+ & \left.\mathcal{H}^{g}(Q, \mu) D_{h / g}\left(z_{h}, k_{T}, \mu, \nu\right) \mathcal{S}_{g}\left(\lambda_{T}, \mu, \nu\right)\right] .
\end{aligned}
$$

Here $D_{h / i}\left(z_{h}, k_{T}, \mu, \nu\right)$ is the usual TMD FF with $k_{T}$ the transverse momentum of the hadron $h$ with respect to the fragmenting parton $i$. On the other hand, $\mathcal{S}_{i}\left(\lambda_{T}, \mu, \nu\right)$ is the soft function, with $\mu$ and $\nu$ renormalization and rapidity scales, respectively, and we have $\mathcal{S}_{\bar{q}}\left(\lambda_{T}, \mu, \nu\right)=\mathcal{S}_{q}\left(\lambda_{T}, \mu, \nu\right)$. The leading-order (LO) cross section is given by

$$
\sigma_{0}=\frac{4 \pi N_{c} \alpha_{\mathrm{em}}^{2}}{3 Q^{2}}
$$

with $\alpha_{\mathrm{em}}$ the fine structure constant. Note that the factorization in eq. (2.3) neglects the power corrections from the ratios $j_{T}^{2} / Q^{2}$, which is small in the kinematic $j_{T} \ll Q$ region we consider. Nevertheless, in the region of $j_{T} \sim Q$ one can include such power corrections from the fixed-order calculations [60]. This is usually referred to as the $Y$-term in the CSS formalism $[28,29]$.

It is important to emphasize that the above TMD formalism is already different from the earlier conjectures used in $[49,50]$. In particular, at leading power, our formalism depends on both quark and gluon TMD FFs, while the previous conjecture contains only quark TMD FFs. To convince that this has to be the case, the easiest way is to look at the Feynman diagram configurations that contribute to our observable at the next-to-leading order (NLO), from which we also derive the hard functions $H^{i}$ with $i=q(\bar{q}), g$. At LO, we produce back-to-back quark $q$ and anti-quark $\bar{q}$, each in their corresponding left or right hemisphere, and our hard function is normalized to be $H=1$ at this order. At NLO, we receive three contributions as shown in figure 2. Here, figure 2(a) is the virtual correction to the LO process $e^{+} e^{-} \rightarrow q \bar{q}$ and $q$ (or $\bar{q}$ ) later on fragments into the observed hadron $h$, and thus this contribution is associated with the quark TMD FFs $D_{h / q}\left(z_{h}, k_{T}, \mu, \nu\right)$ in 
eq. (2.3). Figure 2(b) and 2(c) describe the hard scattering with three partons in the final state, where two hard partons are emitted in the left hemisphere and one parton $i$ in the right hemisphere. Here, for three-particle final states, the thrust axis $\hat{n}$ is determined by the direction of the most energetic parton. For (b), it is $\bar{q}$ and $g$ on the left hemisphere, while $q$ on the right hemisphere, which fragments into the hadron $h$ and thus we have quark TMD FF $D_{h / q}$. For (c), it is $q$ and $\bar{q}$ on the left hemisphere, while $g$ on the right hemisphere which fragments into the hadron $h$ and thus we have gluon TMD FF $D_{h / g}$ in eq. (2.3). We emphasize again that no hard radiation is allowed in the right hemisphere to maintain $j_{T} \ll Q$.

Direct calculations give us the following expressions for the corresponding bare hard functions at NLO [58],

$$
\begin{aligned}
\mathcal{H}_{2}^{q}=\mathcal{H}_{2}^{\bar{q}} \equiv \mathcal{H}_{(a)}^{q}(Q, \epsilon)= & 1+\frac{\alpha_{s}}{4 \pi} C_{F}\left(\frac{\mu^{2}}{Q^{2}}\right)^{\epsilon}\left[-\frac{4}{\epsilon^{2}}-\frac{6}{\epsilon}-16+\frac{7}{3} \pi^{2}\right] \\
\mathcal{H}_{3}^{q}=\mathcal{H}_{3}^{\bar{q}} \equiv \mathcal{H}_{(b)}^{q}(Q, \epsilon)= & \frac{\alpha_{s}}{4 \pi} C_{F}\left(\frac{\mu^{2}}{Q^{2}}\right)^{\epsilon}\left[\frac{2}{\epsilon^{2}}+\frac{3}{\epsilon}+\frac{29}{3}-\frac{3 \pi^{2}}{2}-2 \ln ^{2}(2)\right. \\
& \left.+\frac{5 \ln (3)}{4}-4 \operatorname{Li}_{2}\left(-\frac{1}{2}\right)\right] \\
\mathcal{H}_{3}^{g} \equiv \mathcal{H}_{(c)}^{g}(Q, \epsilon)=\frac{\alpha_{s}}{4 \pi} C_{F} & {\left[-\frac{1}{6}+\frac{\pi^{2}}{3}+2 \ln ^{2}(2)-\frac{5 \ln (3)}{4}+4 \operatorname{Li}_{2}\left(-\frac{1}{2}\right)\right] }
\end{aligned}
$$

where we use the notation $\mathcal{H}_{m}^{i}$ with index $m=2,3$ at NLO representing the number of final-state partons, while the subscripts $(a),(b),(c)$ correspond to the configurations in figure 2. We include the LO result into $\mathcal{H}_{2}^{i}$, and we note that $\mathcal{H}_{3}^{g}$ starts at $\mathcal{O}\left(\alpha_{s}\right)$ order, which is free of any divergence. Note that the function $\mathcal{H}_{2}^{q}$ is the standard dijet hard function, that arises in e.g. back-to-back hadron pair production [17]. If one ignores the non-global structure, the renormalization group (RG) equation for the hard function can be easily obtained from the above expressions. However, the structure for the full RG equations is much more complicated and will be shown in the next section.

\subsection{TMD formalism in coordinate space}

TMD formalism in eq. (2.3) involves convolution over the transverse momentum $\vec{k}_{T}$ and $\vec{\lambda}_{T}$. We apply the Fourier transform to go into the coordinate $b$-space and thus the convolution becomes a simple product. To get started, realizing

$$
\delta^{(2)}\left(\vec{j}_{T}-\vec{k}_{T}-z_{h} \vec{\lambda}_{T}\right)=\frac{1}{z_{h}^{2}} \int \frac{d^{2} \vec{b}}{(2 \pi)^{2}} e^{i \vec{b} \cdot\left(\vec{j}_{T} / z_{h}-\vec{k}_{T} / z_{h}-\vec{\lambda}_{T}\right)}
$$

and we thus can write eq. (2.3) in the following form

$$
\frac{d \sigma}{d z_{h} d^{2} \vec{j}_{T}}=\sigma_{0} \sum_{i=q, \bar{q}, g} e_{q}^{2} \int \frac{d^{2} \vec{b}}{(2 \pi)^{2}} e^{i \vec{b} \cdot \vec{j}_{T} / z_{h}} \mathcal{H}^{i}(Q, \mu) D_{h / i}\left(z_{h}, b, \mu, \nu\right) \mathcal{S}_{i}(b, \mu, \nu)
$$


where the $b$-space TMD FF and soft function are defined as

$$
\begin{aligned}
D_{h / i}\left(z_{h}, b, \mu, \nu\right) & =\frac{1}{z_{h}^{2}} \int d^{2} \vec{k}_{T} e^{-i \vec{b} \cdot \vec{k}_{T} / z_{h}} D_{h / i}\left(z_{h}, k_{T}, \mu, \nu\right), \\
\mathcal{S}_{i}(b, \mu, \nu) & =\int d^{2} \vec{\lambda}_{T} e^{-i \vec{b} \cdot \vec{\lambda}_{T}} \mathcal{S}_{i}\left(\lambda_{T}, \mu, \nu\right) .
\end{aligned}
$$

Both TMD FFs and the soft function suffer from rapidity divergence, which was regularized via the rapidity regulator in $[46,61]$. As a consequence we have rapidity poles in $1 / \eta$ and the associated rapidity scale $\nu$, besides the usual poles in $1 / \epsilon$ in dimensional regularization and the associated renormalization scale $\mu$. In order to resum relevant logarithms, one can use transitional CSS formalism [28], or effective theory approaches [45, 46, 61]. ${ }^{3}$ The NLO perturbative expressions for TMD FFs are well-known [23], and we list here for completeness:

$$
\begin{aligned}
D_{q / q}\left(z_{h}, b, \mu, \nu\right)= & \frac{1}{z_{h}^{2}}\left\{\delta\left(1-z_{h}\right)\right. \\
& +\frac{\alpha_{s}}{2 \pi} C_{F}\left[\frac{2}{\eta}\left(\frac{1}{\epsilon}+\ln \left(\frac{\mu^{2}}{\mu_{b}^{2}}\right)\right)+\frac{1}{\epsilon}\left(\ln \left(\frac{\nu^{2}}{Q^{2}}\right)+\frac{3}{2}\right)\right] \delta\left(1-z_{h}\right) \\
& +\frac{\alpha_{s}}{2 \pi}\left[-\frac{1}{\epsilon}-\ln \left(\frac{\mu^{2}}{z_{h}^{2} \mu_{b}^{2}}\right)\right] P_{q q}\left(z_{h}\right) \\
& \left.+\frac{\alpha_{s}}{2 \pi} C_{F}\left[\ln \left(\frac{\mu^{2}}{\mu_{b}^{2}}\right)\left(\ln \left(\frac{\nu^{2}}{Q^{2}}\right)+\frac{3}{2}\right) \delta\left(1-z_{h}\right)+\left(1-z_{h}\right)\right]\right\} \\
D_{g / q}\left(z_{h}, b, \mu, \nu\right)= & \frac{1}{z_{h}^{2}}\left\{\frac{\alpha_{s}}{2 \pi}\left[-\frac{1}{\epsilon}-\ln \left(\frac{\mu^{2}}{z_{h}^{2} \mu_{b}^{2}}\right)\right] P_{g q}\left(z_{h}\right)+\frac{\alpha_{s}}{2 \pi} C_{F} z_{h}\right\},
\end{aligned}
$$

where the splitting functions are given by

$$
P_{q q}\left(z_{h}\right)=C_{F}\left[\frac{1+z_{h}^{2}}{\left(1-z_{h}\right)_{+}}+\frac{3}{2} \delta\left(1-z_{h}\right)\right], \quad P_{g q}\left(z_{h}\right)=C_{F} \frac{1+\left(1-z_{h}\right)^{2}}{z_{h}} .
$$

The NLO soft function $S_{q}(b, \mu, \nu)$ can also be computed easily. Since at NLO, only the soft radiation that is emitted in the right hemisphere contributes to the hadron transverse momentum $j_{T}$, this will put a constraint for the soft gluon momentum $k$ in the soft function, i.e., $k_{z}>0$ or $k^{-}>k^{+}$.

$$
\begin{aligned}
\mathcal{S}_{q}(b, \mu, \nu)= & \int d^{2} \vec{\lambda}_{T} e^{-i \vec{\lambda}_{T} \cdot \vec{b}}\left[\delta^{2}\left(\vec{\lambda}_{T}\right)+\frac{\alpha_{s} C_{F}}{2 \pi^{2}} \frac{e^{\epsilon \gamma_{E}}}{\Gamma(1-\epsilon)} \int \frac{d k^{+} d k^{-}}{2}\right. \\
& \left.\times\left(\frac{\mu^{2}}{\vec{\lambda}_{T}^{2}}\right)^{\epsilon} \frac{2 n \cdot \bar{n}}{k^{+} k^{-}} \delta^{+}\left(k^{+} k^{-}-\vec{\lambda}_{T}^{2}\right)\left|\frac{\nu}{2 k_{z}}\right|^{\eta} \theta\left(1-\frac{k^{+}}{k^{-}}\right)\right] \\
= & 1+\frac{\alpha_{s}}{2 \pi} C_{F}\left[\frac{2}{\eta}\left(-\frac{1}{\epsilon}-\ln \left(\frac{\mu^{2}}{\mu_{b}^{2}}\right)\right)+\frac{1}{\epsilon^{2}}-\frac{1}{\epsilon} \ln \left(\frac{\nu^{2}}{\mu^{2}}\right)\right. \\
& \left.-\ln \left(\frac{\mu^{2}}{\mu_{b}^{2}}\right) \ln \left(\frac{\nu^{2}}{\mu_{b}^{2}}\right)+\frac{1}{2} \ln ^{2}\left(\frac{\mu^{2}}{\mu_{b}^{2}}\right)-\frac{\pi^{2}}{12}\right] .
\end{aligned}
$$

\footnotetext{
${ }^{3}$ We recommend [62] for the comparison for different TMD factorization frameworks.
} 
It might be instructive to point out that the above soft function is exactly half of the standard soft function for the back-to-back hadron pair production in $e^{+} e^{-}$collisions, as well as those in SIDIS and Drell-Yan processes. This difference is precisely introduced by the constraint $k_{z}>0$ for the radiated soft gluon. This situation is similar to the case where one measures the transverse momentum of hadrons inside a jet with a jet radius $R$, as studied in [23], where soft functions in these two situations are related to each other by a boost along the $z$-direction.

With the explicit expressions for TMD FFs and soft function at NLO given above, one can easily obtain their corresponding $\mu$ and $\nu$ evolution equations:

$$
\begin{aligned}
\frac{d}{d \ln \mu} \ln D_{h / q}\left(z_{h}, b, \mu, \nu\right) & =\gamma_{\mu}^{D}\left(\alpha_{s}\right), \\
\frac{d}{d \ln \nu} \ln D_{h / q}\left(z_{h}, b, \mu, \nu\right) & =\gamma_{\nu}^{D}\left(\alpha_{s}\right), \\
\frac{d}{d \ln \mu} \ln \mathcal{S}_{q}(b, \mu, \nu) & =\gamma_{\mu}^{S}\left(\alpha_{s}\right), \\
\frac{d}{d \ln \nu} \ln \mathcal{S}_{q}(b, \mu, \nu) & =\gamma_{\nu}^{S}\left(\alpha_{s}\right) .
\end{aligned}
$$

Here the relevant anomalous dimensions are given by

$$
\begin{aligned}
& \gamma_{\mu}^{D}\left(\alpha_{s}\right)=\Gamma_{\text {cusp }}\left(\alpha_{s}\right) \ln \left(\frac{\nu^{2}}{Q^{2}}\right)+2 \gamma^{D_{q}}\left(\alpha_{s}\right), \\
& \gamma_{\mu}^{S}\left(\alpha_{s}\right)=-\Gamma_{\text {cusp }}\left(\alpha_{s}\right) \ln \left(\frac{\nu^{2}}{\mu^{2}}\right)+\gamma^{S}\left(\alpha_{s}\right), \\
& \gamma_{\nu}^{D}\left(\alpha_{s}\right)=-\gamma_{\nu}^{S}\left(\alpha_{s}\right)=\Gamma_{\text {cusp }}\left(\alpha_{s}\right) \ln \left(\frac{\mu^{2}}{\mu_{b}^{2}}\right),
\end{aligned}
$$

where the cusp anomalous dimensions $\Gamma_{\text {cusp }}$ and the non-cusp $\gamma^{D_{q}, S}$ have their usual expansion

$$
\Gamma_{\text {cusp }}\left(\alpha_{s}\right)=\sum_{n=1} \Gamma_{n-1}\left(\frac{\alpha_{s}}{4 \pi}\right)^{n}, \quad \gamma^{D_{q}, S}=\sum_{n=1} \gamma_{n-1}^{D_{q}, S}\left(\frac{\alpha_{s}}{4 \pi}\right)^{n}
$$

We have the first few coefficients given by

$$
\begin{aligned}
\Gamma_{0} & =4 C_{F}, & \Gamma_{1} & =\left(\frac{268}{9}-\frac{4 \pi^{2}}{3}\right) C_{F} C_{A}-\frac{40}{9} C_{F}^{2} n_{f}, \\
\gamma_{0}^{D_{q}} & =3 C_{F}, & \gamma_{0}^{S} & =0
\end{aligned}
$$

where $C_{F}=\left(N_{c}^{2}-1\right) /\left(2 N_{c}\right)$ with $N_{c}=3, C_{A}=3$, and $n_{f}$ represents the quark flavor number.

It is important to realize that the rapidity divergences between TMD FF $D_{h / q}\left(z_{h}, b, \mu, \nu\right)$ and soft function $\mathcal{S}_{q}(b, \mu, \nu)$ cancel between them. This is to be compared with the standard case, e.g., back-to-back hadron pair production in $e^{+} e^{-}$collisions, where the rapidity 
divergences cancel between one TMD FF $D_{h / q}\left(z_{h}, b, \mu, \nu\right)$ and the square-root of the standard soft function $\sqrt{S_{q}(b, \mu, \nu)}$, see e.g. [17, 29, 61, 62]. Following the modern formulation of TMD FFs, we combine them as the so-called properly-defined TMD FFs [29,62] as follows:

$$
\mathcal{D}_{h / q}^{\mathrm{TMD}}\left(z_{h}, b, \mu\right)=D_{h / q}\left(z_{h}, b, \mu, \nu\right) \mathcal{S}_{q}(b, \mu, \nu)
$$

Using the evolution equations in eq. (2.14), one can obtain the evolved TMD FFs $\mathcal{D}^{\mathrm{TMD}}$ to be at the hard scale $\mu_{h} \sim Q$ and thus resum the relevant logarithms $\sim \ln \left(Q^{2} / j_{T}^{2}\right)$. For example, the standard exercise is to evolve $\mathcal{S}_{q}$ from its characteristic scales $\mu_{s} \sim \mu_{b}$ and $\nu_{s} \sim \mu_{b}$, and $D_{h / q}$ from its natural scales $\mu_{D} \sim \mu_{b}$ and $\nu_{D} \sim Q$, to the hard scale $\mu_{h} \sim Q$ and a common rapidity scale $\nu$, from which one obtains

$$
\begin{aligned}
D_{h / q}\left(z_{h}, b, \mu_{h}, \nu\right)= & D_{h / q}\left(z_{h}, b, \mu_{b}, \nu_{D}\right)\left(\frac{\nu}{\nu_{D}}\right)^{-K\left(b, \mu_{b}\right)} \\
& \times \exp \left\{\int_{\mu_{b}}^{\mu_{h}} \frac{d \mu}{\mu}\left[\Gamma_{\text {cusp }}\left(\alpha_{s}\right) \ln \left(\frac{\nu^{2}}{Q^{2}}\right)+2 \gamma^{D_{q}}\left(\alpha_{s}\right)\right]\right\}, \\
\mathcal{S}_{q}\left(b, \mu_{h}, \nu\right)= & \mathcal{S}_{q}\left(b, \mu_{b}, \nu_{s}\right)\left(\frac{\nu}{\nu_{s}}\right)^{K\left(b, \mu_{b}\right)} \\
& \times \exp \left\{\int_{\mu_{b}}^{\mu_{h}} \frac{d \mu}{\mu}\left[-\Gamma_{\text {cusp }}\left(\alpha_{s}\right) \ln \left(\frac{\nu^{2}}{\mu^{2}}\right)+\gamma^{S}\left(\alpha_{s}\right)\right]\right\},
\end{aligned}
$$

where $K\left(b, \mu_{b}\right)=\left.\gamma_{\nu}^{S}\left(\alpha_{s}\right)\right|_{\mu=\mu_{b}}$ is the rapidity anomalous dimension [46, 61] or Collins-Soper kernel $[29,62]$. Combine the above evolution equations, we thus obtain

$$
\mathcal{D}_{h / q}^{\mathrm{TMD}}\left(z_{h}, b, \mu_{h}\right)=\mathcal{D}_{h / q}^{\mathrm{TMD}}\left(z_{h}, b, \mu_{b}\right) e^{-S_{\mathrm{pert}}\left(\mu_{b}, \mu_{h}\right)}\left(\frac{\nu_{D}}{\nu_{s}}\right)^{K\left(b, \mu_{b}\right)}
$$

where we have

$$
\begin{aligned}
& \mathcal{D}_{h / q}^{\mathrm{TMD}}\left(z_{h}, b, \mu_{h}\right)=D_{h / q}\left(z_{h}, b, \mu_{h}, \nu\right) \mathcal{S}_{q}\left(b, \mu_{h}, \nu\right), \\
& \mathcal{D}_{h / q}^{\mathrm{TMD}}\left(z_{h}, b, \mu_{b}\right)=D_{h / q}\left(z_{h}, b, \mu_{b}, \nu_{D}\right) \mathcal{S}_{q}\left(b, \mu_{b}, \nu_{s}\right) .
\end{aligned}
$$

On the other hand, the exponent of the evolution factor, i.e. the perturbative Sudakov factor $S_{\text {pert }}\left(\mu_{b}, \mu_{h}\right)$ resums all the global logarithms and is given by

$$
S_{\text {pert }}\left(\mu_{b}, \mu_{h}\right)=\int_{\mu_{b}}^{\mu_{h}} \frac{d \mu}{\mu}\left[\Gamma_{\text {cusp }}\left(\alpha_{s}\right) \ln \left(\frac{Q^{2}}{\mu^{2}}\right)-2 \gamma^{D_{q}}\left(\alpha_{s}\right)-\gamma^{S}\left(\alpha_{s}\right)\right] .
$$

Finally when the scale $\mu_{b} \gg \Lambda_{\mathrm{QCD}}$, one can further match the TMD FFs $\mathcal{D}_{h / q}^{\mathrm{TMD}}\left(z_{h}, b, \mu_{b}\right)$ onto the collinear FFs $D_{h / q}\left(z_{h}, \mu_{b}\right)$ :

$$
\mathcal{D}_{h / q}^{\mathrm{TMD}}\left(z_{h}, b, \mu_{b}\right)=\frac{1}{z_{h}^{2}} \sum_{i} \int_{z_{h}}^{1} \frac{d z}{z} C_{i \leftarrow q}\left(z, b, \mu_{b}\right) D_{h / i}\left(z_{h} / z, \mu_{b}\right)+\mathcal{O}\left(\frac{\Lambda_{\mathrm{QCD}}^{2}}{\mu_{b}^{2}}\right)
$$


where $C_{i \leftarrow q}\left(z, \mu_{b}\right)=\delta_{i q} \delta(1-z)$ at LO and the higher-order expressions can be found in $[17,29,63,64]$.

On the other hand, when $\mu_{b} \sim \Lambda_{\mathrm{QCD}}$, one has to introduce non-perturbative contributions, for which we apply the usual $b_{*}$-prescription to include the TMD evolution in the large $b$ region. Here we have $b_{*}$ defined as

$$
b_{*}=\frac{b}{\sqrt{1+b^{2} / b_{\max }^{2}}}
$$

where $b_{\max }$ is chosen [17] to be $1.5 \mathrm{GeV}^{-1}$. At the same time we include non-perturbative function $S_{\mathrm{NP}}\left(b, Q_{0}, Q\right)$, which is given by $[23,36]$

$$
S_{\mathrm{NP}}\left(b, Q_{0}, Q\right)=\frac{g_{2}}{2} \ln \left(\frac{b}{b_{*}}\right) \ln \left(\frac{Q}{Q_{0}}\right)+\frac{g_{h}}{z_{h}^{2}} b^{2},
$$

with $Q_{0}^{2}=2.4 \mathrm{GeV}^{2}, g_{2}=0.84$ and $g_{h}=0.042$. We choose to work at the next-toleading logarithmic (NLL) level, we thus include two-loop cusp and one-loop normal anomalous dimension, and tree-level matching coefficients. Then plugging in the above results for $\mathcal{D}_{h / q}^{\mathrm{TMD}}\left(z_{h}, b, \mu_{h}\right)$ in eqs. (2.23) and (2.27), along with the non-perturbative function $S_{\mathrm{NP}}\left(b, Q_{0}, Q\right)$ in eq. (2.29), into the differential cross section in eq. (2.8), we obtain the all-order resummation formula

$$
\frac{d \sigma}{d z_{h} d^{2} \vec{j}_{T}}=\sigma_{0} \sum_{i=q, \bar{q}} e_{i}^{2} \int_{0}^{\infty} \frac{b d b}{2 \pi} J_{0}\left(b j_{T} / z_{h}\right) e^{-S_{\mathrm{pert}}\left(\mu_{b *}, \mu_{h}\right)-S_{\mathrm{NP}}\left(b, Q_{0}, Q\right)} \frac{1}{z_{h}^{2}} D_{h / i}\left(z_{h}, \mu_{b *}\right),
$$

where the Bessel function $J_{0}$ arises after integrating the angle between $\vec{b}$ and $\vec{j}_{T}$. The numerical integration over $b$ is performed using the algorithm in [65]. We have chosen the canonical scales for $\mu_{h}$ and $\mu_{b_{*}}$ as follows

$$
\mu_{h}=Q, \quad \mu_{b *}=2 e^{-\gamma_{E}} / b_{*} .
$$

Such a formalism in eq. (2.30) resums all the global logarithms in $\ln \left(Q^{2} / j_{T}^{2}\right)$.

\subsection{TMD formalism at threshold $z_{h} \rightarrow 1$}

Belle collaboration finds that the hadron cross sections can be well described by Gaussians Distribution as a function of $j_{T}$ in the small $j_{T}$ region and that the width of the Gaussian shows an initially rising for small to intermediate $z_{h}$, while a decreasing $z_{h}$-dependence for large $z_{h} \lesssim 1$. In the region $z_{h} \rightarrow 1$ region, the threshold logarithms $\ln \left(1-z_{h}\right)$ would become important and thus has to be resummed. In our phenomenological section, we find that the joint threshold and TMD resummation will be able to describe well such a $z_{h}$-dependence for the Gaussian width. We develop theoretical formalism in this section for this purpose. As we will show below, in the threshold region, an additional mode, so-called collinear-soft (c-soft) mode [66-69] is relevant. Such a mode is shown as the purple curves in figure 1 (right), and the corresponding momentum scaling is given by

- c-soft: $\quad p_{\mathscr{S}} \sim\left(j_{T}^{2} /\left(Q\left(1-z_{h}\right)\right), Q\left(1-z_{h}\right), j_{T}\right)$. 
Let us start our discussion with the fixed-order result of the perturbative TMD FFs $D_{q / q}$ and $D_{g / q}$ in eq. (2.11) in the threshold limit. By taking the limit $z_{h} \rightarrow 1$, we find at NLO

$$
\begin{aligned}
D_{q / q}\left(z_{h}, b, \mu, \nu\right)= & \frac{1}{z_{h}^{2}}\left\{\delta\left(1-z_{h}\right)\right. \\
& +\frac{\alpha_{s}}{2 \pi} C_{F}\left[\frac{2}{\eta}\left(\frac{1}{\epsilon}+\ln \left(\frac{\mu^{2}}{\mu_{b}^{2}}\right)\right)+\frac{1}{\epsilon}\left(\ln \left(\frac{\nu^{2}}{Q^{2}}\right)+\frac{3}{2}\right)\right] \delta\left(1-z_{h}\right) \\
& +\frac{\alpha_{s}}{2 \pi} C_{F}\left[-\frac{1}{\epsilon}-\ln \left(\frac{\mu^{2}}{\mu_{b}^{2}}\right)\right]\left[\frac{2}{\left(1-z_{h}\right)_{+}}+\frac{3}{2} \delta\left(1-z_{h}\right)\right] \\
& \left.+\frac{\alpha_{s}}{2 \pi} C_{F}\left[\ln \left(\frac{\mu^{2}}{\mu_{b}^{2}}\right)\left(\ln \left(\frac{\nu^{2}}{Q^{2}}\right)+\frac{3}{2}\right) \delta\left(1-z_{h}\right)\right]\right\}
\end{aligned}
$$

where we keep the overall factor of $1 / z_{h}^{2}$ as a convention. Note that in this limit, one can drop the mixing term $D_{g / q}$ in comparison with the more singular terms $\delta\left(1-z_{h}\right)$ and $\frac{1}{\left(1-z_{h}\right)_{+}}$in $D_{q / q}$, i.e. only the flavor diagonal $q \rightarrow q$ channel contributes. In the threshold limit, we can refactorize the TMD FF $D_{h / q}$ as

$$
D_{h / q}\left(z_{h}, b, \mu, \nu\right)=\int_{z_{h}}^{1} \frac{d z}{z} \mathscr{S}_{q}(z, b, \mu, \nu) D_{h / q}\left(z_{h} / z, \mu\right),
$$

where $\mathscr{S}_{q}$ is a collinear-soft (c-soft) function [66-69] that takes into account the soft radiation along the direction of the thrust axis, i.e. the c-soft mode mentioned above. At NLO, it can be computed as follows

$$
\begin{aligned}
\mathscr{S}_{q}(z, b, \mu, \nu)= & \delta(1-z)+\frac{\alpha_{s} C_{F}}{2 \pi^{2}} \frac{e^{\epsilon \gamma_{E}}}{\Gamma(1-\epsilon)} \int \frac{d k^{+} d k^{-}}{2} \int d^{2} \vec{k}_{T} e^{-i \vec{k}_{T} \cdot \vec{b}} \frac{1}{\mu^{2}} \\
& \times\left(\frac{\mu^{2}}{\vec{k}_{T}^{2}}\right)^{1+\epsilon} \frac{2 n \cdot \bar{n}}{k^{+} k^{-}} \delta^{+}\left(k^{2}\right) \delta\left(k^{-}-(1-z) Q\right)\left|\frac{\nu}{2 k_{z}}\right|^{\eta} \\
= & \delta(1-z)+\frac{\alpha_{s}}{2 \pi} C_{F}\left[\frac{1}{\epsilon}+\ln \left(\frac{\mu^{2}}{\mu_{b}^{2}}\right)\right]\left[\left(\frac{2}{\eta}+\ln \left(\frac{\nu^{2}}{Q^{2}}\right)\right) \delta(1-z)-\frac{2}{(1-z)_{+}}\right] .
\end{aligned}
$$

Note that the c-soft function $\mathscr{S}_{q}(z, b, \mu, \nu)$ has the same rapidity anomalous dimension as the TMD FF $D_{h / q}\left(z_{h}, b, \mu, \nu\right)$, which is cancelled after combining soft function $\mathcal{S}_{q}$ in eq. (2.13) and the c-soft function in eq. (2.34). On the other hand, we also have the collinear FFs at the threshold limit, whose perturbative expression is given by

$$
D_{q / q}\left(z_{h}, \mu\right)=\delta\left(1-z_{h}\right)+\frac{\alpha_{s}}{2 \pi} C_{F}\left(-\frac{1}{\epsilon}\right)\left[\frac{2}{\left(1-z_{h}\right)_{+}}+\frac{3}{2} \delta\left(1-z_{h}\right)\right] .
$$

To perform the resummation in the threshold limit, one can perform the Mellin transform or Laplace transformation [70], whose purpose is to convert the above convolution in $z$-space into a simple product in the corresponding transformed space. Here we choose to perform the Laplace transformation [71],

$$
\tilde{D}_{h / q}(\kappa, b, \mu, \nu)=\int_{0}^{\infty} d \bar{z}_{h} e^{-\frac{\bar{z}_{h}}{\kappa e^{\gamma} E}} D_{h / q}\left(1-\bar{z}_{h}, b, \mu, \nu\right),
$$


where $\bar{z}_{h}=1-z_{h}$. Using the following relation in the threshold limit

$$
1-z_{h}=1-\left[1-\left(1-\frac{z_{h}}{z}\right)\right][1-(1-z)] \approx\left(1-\frac{z_{h}}{z}\right)+(1-z)
$$

one can express eq. (2.33) as a product in the Laplace space

$$
\tilde{D}_{h / q}(\kappa, b, \mu, \nu)=\tilde{\mathscr{S}}_{q}(\kappa, b, \mu, \nu) \tilde{D}_{h / q}(\kappa, \mu) .
$$

Note that we have also extended the integration from $0<\bar{z}<1$ to $0<\bar{z}<\infty$ in the threshold limit approximation. The NLO expressions for $\tilde{\mathscr{S}}_{q}(\kappa, b, \mu, \nu)$ and $\tilde{D}_{h / q}(\kappa, \mu)$ in the Laplace space are given by

$$
\begin{aligned}
\tilde{\mathscr{S}}_{q}(\kappa, b, \mu, \nu) & =1+\frac{\alpha_{s}}{2 \pi} C_{F}\left[\frac{1}{\epsilon}+\ln \left(\frac{\mu^{2}}{\mu_{b}^{2}}\right)\right]\left[\frac{2}{\eta}+\ln \left(\frac{\nu^{2}}{\kappa^{2} Q^{2}}\right)\right], \\
\tilde{D}_{q / q}(\kappa, \mu) & =1+\frac{\alpha_{s}}{2 \pi} C_{F}\left(-\frac{1}{\epsilon}\right)\left[\ln \left(\kappa^{2}\right)+\frac{3}{2}\right] .
\end{aligned}
$$

From the above results, one can derive the RG equations for both $\tilde{\mathscr{S}}_{q}$ and $D_{q / q}$ in the Laplace space,

$$
\begin{aligned}
\frac{d}{d \ln \mu} \ln \tilde{\mathscr{S}}_{q}(\kappa, b, \mu, \nu) & =\left[\Gamma_{\text {cusp }}\left(\alpha_{s}\right) \ln \left(\frac{\nu^{2}}{\kappa^{2} Q^{2}}\right)+\gamma^{\tilde{\mathscr{S}}_{q}}\left(\alpha_{s}\right)\right], \\
\frac{d}{d \ln \nu} \ln \tilde{\mathscr{S}}_{q}(\kappa, b, \mu, \nu) & =\gamma_{\nu}^{D}\left(\alpha_{s}\right), \\
\frac{d}{d \ln \mu} \ln \tilde{D}_{h / q}(\kappa, \mu) & =\left[\Gamma_{\text {cusp }}\left(\alpha_{s}\right) \ln \left(\kappa^{2}\right)+2 \gamma^{f_{q}}\left(\alpha_{s}\right)\right]
\end{aligned}
$$

where the normal anomalous dimensions $\gamma^{i}$ expanded as $\gamma^{i}=\sum_{n=1} \gamma_{n-1}^{i}\left(\alpha_{s} / 4 \pi\right)^{n}$ with $i=\tilde{\mathscr{S}}_{q}, f_{q}$, and

$$
\gamma_{0}^{f_{q}}=3 C_{F}, \quad \gamma_{0}^{\tilde{\mathscr{I}} q}=0
$$

The above RG equations allow us to evolve c-soft function $\tilde{\mathscr{S}}_{q}(\kappa, b, \mu, \nu)$ from its natural scale $\mu_{\mathscr{S}} \sim \mu_{b}$ and $\nu_{\mathscr{S}} \sim \kappa Q$, and the FF $D_{h / q}(\kappa, \mu)$ from initial scale $\mu_{F}$, up to the hard scale $\mu_{h}$ and a rapidity scale $\nu$, we obtain

$$
\begin{aligned}
\tilde{\mathscr{S}}_{q}\left(\kappa, b, \mu_{h}, \nu\right)= & \tilde{\mathscr{S}}_{q}\left(\kappa, b, \mu_{b}, \nu \mathscr{S}\right)\left(\frac{\nu}{\nu_{\mathscr{S}}}\right)^{-K\left(b, \mu_{b}\right)} \\
& \times \exp \left\{\int_{\mu_{b}}^{\mu_{h}} \frac{d \mu}{\mu}\left[\Gamma_{\text {cusp }}\left(\alpha_{s}\right) \ln \left(\frac{\nu^{2}}{\kappa^{2} Q^{2}}\right)+\gamma^{\tilde{\mathscr{S}}_{q}}\left(\alpha_{s}\right)\right]\right\}, \\
D_{h / q}\left(\kappa, \mu_{h}\right)= & D_{h / q}\left(\kappa, \mu_{F}\right) \exp \left[\int_{\mu_{F}}^{\mu_{h}} \frac{d \mu}{\mu}\left(\Gamma_{\text {cusp }}\left(\alpha_{s}\right) \ln \left(\kappa^{2}\right)+2 \gamma^{f_{q}}\left(\alpha_{s}\right)\right)\right] .
\end{aligned}
$$

Combining the evolution for the global soft function $S_{q}(b, \mu, \nu)$ in eq. (2.22), we obtain the following evolution for the properly-defined TMD FFs in the Laplace space,

$$
\begin{aligned}
\tilde{\mathcal{D}}_{h / q}^{\mathrm{TMD}}\left(\kappa, b, \mu_{h}\right)= & S_{q}\left(b, \mu_{b}, \nu_{s}\right) \tilde{\mathscr{S}}_{q}\left(\kappa, b, \mu_{b}, \nu_{\mathscr{S}}\right) \tilde{D}_{h / q}\left(\kappa, \mu_{F}\right) \\
& \times e^{-\tilde{S}_{\mathrm{pert}}\left(\mu_{b}, \mu_{h}\right)}\left(\frac{\nu_{\mathscr{S}}}{\nu_{s}}\right)^{K\left(b, \mu_{b}\right)} \cdot
\end{aligned}
$$


Here the perturbative Sudakov factor $\tilde{S}_{\text {pert }}\left(\mu_{b}, \mu_{h}\right)$ is given by

$$
\begin{aligned}
\tilde{S}_{\text {pert }}\left(\mu_{b}, \mu_{h}\right)= & \int_{\mu_{b}}^{\mu_{h}} \frac{d \mu}{\mu}\left[\Gamma_{\text {cusp }}\left(\alpha_{s}\right) \ln \left(\frac{\kappa^{2} Q^{2}}{\mu^{2}}\right)\right] \\
& -\int_{\mu_{F}}^{\mu_{h}} \frac{d \mu}{\mu}\left[\Gamma_{\text {cusp }}\left(\alpha_{s}\right) \ln \left(\kappa^{2}\right)+2 \gamma^{f_{q}}\left(\alpha_{s}\right)\right],
\end{aligned}
$$

where the first integral represents the evolution of c-soft function and the global part of the soft function from $\mu_{b}$ to $\mu_{h}$, and the second one is the collinear fragmentation function from factorization scale $\mu_{F}$ to $\mu_{h}$ in the threshold limit. Performing the inverse Laplace transform, we obtain the following expression for TMD FFs in the threshold limit

$$
\mathcal{D}_{h / q}^{\mathrm{TMD}}\left(z_{h}, b, \mu_{h}\right)=\frac{1}{z_{h}^{2}} \int_{z_{h}}^{1} \frac{d z}{z} e^{-\hat{S}_{\mathrm{pert}}\left(\mu_{b}, \mu_{h}\right)} \frac{e^{-2 \gamma_{E} \eta}}{\Gamma(2 \eta)} \frac{1}{1-z} D_{h / q}\left(z_{h} / z, \mu_{h}\right) .
$$

Here we derive the above formula using the first line of Sudakov factor in eq. (2.48) and setting $\mu_{F}=\mu_{h}$, and the parameter $\eta$ is defined as

$$
\eta=-\int_{\mu_{b}}^{\mu_{h}} \frac{d \mu}{\mu} \Gamma_{\text {cusp }}\left(\alpha_{s}\right)
$$

On the other hand, $\hat{S}_{\text {pert }}\left(\mu_{b}, \mu_{h}\right)$ in the momentum space in the threshold limit is given by

$$
\hat{S}_{\text {pert }}\left(\mu_{b}, \mu_{h}\right)=\int_{\mu_{b}}^{\mu_{h}} \frac{d \mu}{\mu}\left[\Gamma_{\text {cusp }}\left(\alpha_{s}\right) \ln \left(\frac{(1-z)^{2} Q^{2}}{\mu^{2}}\right)\right],
$$

where the argument in the logarithm is given by $(1-z) Q$ in the threshold limit.

Finally using the above result, one can obtain the resummed formalism for the differential cross section at the NLL level

$$
\begin{aligned}
\frac{d \sigma}{d z_{h} d^{2} \vec{j}_{T}}= & \sigma_{0} \sum_{i=q, \bar{q}} \int_{0}^{\infty} \frac{b d b}{2 \pi} J_{0}\left(b j_{T} / z_{h}\right) \\
& \times \frac{1}{z_{h}^{2}} \int_{z_{h}}^{1} \frac{d z}{z} e^{-\hat{S}_{\mathrm{pert}}\left(\mu_{b_{*}}, \mu_{h}\right)-\hat{S}_{\mathrm{NP}}\left(b, Q_{0}, Q\right)} \frac{e^{-2 \gamma_{E} \eta}}{\Gamma(2 \eta)} \frac{1}{1-z} D_{h / i}\left(z_{h} / z, \mu_{h}\right)
\end{aligned}
$$

where we choose the non-perturbative Sudakov factor $\hat{S}_{\mathrm{NP}}\left(b, Q_{0}, Q\right)$ to have the following form

$$
\hat{S}_{\mathrm{NP}}\left(b, Q_{0}, Q\right)=\frac{g_{2}}{2} \ln \left(\frac{b}{b_{*}}\right) \ln \left[\frac{Q\left(1-z_{h}\right)}{Q_{0}}\right]+\frac{g_{h}}{z_{h}^{2}} b^{2}
$$

Here motivated by the argument in the perturbative Sudakov function in eq. (2.51), we replace $Q$ by $\left(1-z_{h}\right) Q$ in the usual non-perturbative function $S_{\mathrm{NP}}\left(b, Q_{0}, Q\right)$ in eq. (2.29) to obtain $\hat{S}_{\mathrm{NP}}\left(b, Q_{0}, Q\right)$ in the threshold limit. 


\section{Factorization and resummation: full story}

The plane perpendicular to the thrust axis divides the full space into two hemispheres. One measures the transverse momentum of hadron $h$ in the right hemisphere, and the left hemisphere is inclusive. As we have emphasized above, hadron transverse momentum with respect to the thrust axis is a non-global observable, since the left hemisphere is unobserved. Such type of observables will involve non-global structures that can not be captured by the traditional exponential formula [52]. In this section, we apply formalism developed in $[58,59]$ to derive the factorization and resummation formula. Such a formalism will enable us to resum both global and non-global logarithms. The global logarithmic structure has been discussed in the previous section. Here in this section, we pay more attention to the NGLs [57]. Very recently, a similar structure is also mentioned in [72].

In the standard TMD region where $j_{T} \ll Q$, following the development in [58, 59], we can write the factorization formalism as follows

$$
\begin{aligned}
\frac{d \sigma}{d z_{h} d^{2} \vec{j}_{T}}= & \sum_{i=q, \bar{q}, g} \int d^{2} \vec{k}_{T} d^{2} \vec{\lambda}_{T} \delta^{(2)}\left(\vec{j}_{T}-\vec{k}_{T}-z_{h} \vec{\lambda}_{T}\right) \\
& \times \sum_{m=2}^{\infty} \frac{1}{N_{c}} \operatorname{Tr}_{c}\left[\mathcal{H}_{m}^{i}(\{\underline{n}\}, Q, \mu) \otimes \mathcal{S}_{m}\left(\{\underline{n}\}, \lambda_{T}, \mu, \nu\right)\right] D_{h / i}\left(z_{h}, k_{T}, \mu, \nu\right),
\end{aligned}
$$

where $\mathcal{H}, \mathcal{S}$, and $D_{h / i}$ correspond to hard, soft and TMD FFs, respectively. Besides, different from the formalism in the previous section that resums only global logarithms, the hard and soft functions are now matrices in the color space, so we take color averaging as $\operatorname{Tr}_{c}[\cdots] / N_{c}$ after multiplying them and integrating out the solid angles $\{\underline{n}\}=\left\{n_{1}, n_{2}, \cdots\right\}$ of the hard partons, where the angular integration is expressed by the symbol $\otimes$. The index $m$ denotes the number of energetic partons inside the hard function that is defined in [54]. The index $m$ in soft function then represents the number of Wilson lines, and the momentum space soft function is defined as

$$
\begin{aligned}
\mathcal{S}_{m}\left(\{\underline{n}\}, \lambda_{T}\right)= & \int \sum_{X_{R}} \delta^{(2)}\left(\vec{p}_{X_{R}}^{\perp}-\vec{\lambda}_{T}\right) \\
& \times\left\langle 0\left|\boldsymbol{S}_{0}^{\dagger}(n) \boldsymbol{S}_{1}^{\dagger}\left(n_{1}\right) \ldots \boldsymbol{S}_{m}^{\dagger}\left(n_{m}\right)\right| X_{R}\right\rangle\left\langle X_{R}\left|\boldsymbol{S}_{0}(n) \boldsymbol{S}_{1}\left(n_{1}\right) \ldots \boldsymbol{S}_{m}\left(n_{m}\right)\right| 0\right\rangle .
\end{aligned}
$$

Here $X_{R}$ denotes soft states in the right hemisphere, and one only measures the contributions from soft radiations in the right hemisphere. It is precise because of such a multiWilson line structure that makes the hard and soft function matrices in the color space. After performing Fourier transformation for the observed $\vec{j}_{T}$, the factorization formula is given as

$$
\begin{aligned}
\frac{d \sigma}{d z_{h} d^{2} \overrightarrow{j_{T}}}= & \sum_{i=q, \bar{q}, g} \int \frac{d^{2} \vec{b}}{(2 \pi)^{2}} e^{i \vec{b} \cdot \vec{j}_{T} / z_{h}} \\
& \times \sum_{m=2}^{\infty} \frac{1}{N_{c}} \operatorname{Tr}_{c}\left[\mathcal{H}_{m}^{i}(\{\underline{n}\}, Q, \mu) \otimes \mathcal{S}_{m}(\{\underline{n}\}, b, \mu, \nu)\right] D_{h / i}\left(z_{h}, b, \mu, \nu\right) .
\end{aligned}
$$


For the NGLs resummation, we use the same methods in [58] to perform renormalization for the multi-Wilson-line operators. The renormalization constants of the hard and soft function are matrices in the color space, which are given as

$$
\begin{aligned}
\mathcal{H}_{m}(\{\underline{n}\}, Q, \epsilon) & =\sum_{l=2}^{m} \mathcal{H}_{l}(\{\underline{n}\}, Q, \mu) \boldsymbol{Z}_{l m}^{H}(\{\underline{n}\}, \mu, \epsilon), \\
\mathcal{S}_{l}(\{\underline{n}\}, b, \mu, \nu) & =\sum_{m=l}^{\infty} \boldsymbol{Z}_{l m}^{S}(\{\underline{n}\}, b, \mu, \nu, \epsilon, \eta) \hat{\otimes} \mathcal{S}_{m}(\{\underline{n}\}, b, \epsilon, \eta),
\end{aligned}
$$

separately. The symbol $\hat{\otimes}$ denotes the integration of the angular vectors $\left\{n_{l+1}, n_{l+2}, \cdots\right\}$ defined in [59]. The factor $\boldsymbol{Z}^{H}$ and $\boldsymbol{Z}^{S}$ are connected through the renormalization factor $\left(Z^{D}\right)$ of the TMD FF as $\boldsymbol{Z}^{S}=Z^{D} \boldsymbol{Z}^{H}$. The above relations have been verified at the two-loop order in $[54,58]$. For convenience, we define the global renormalization constant $Z^{S}$ for the soft function as [11]

$$
\mathcal{S}_{2}(\{\underline{n}\}, b, \epsilon, \eta)=\mathcal{S}_{2}(\{\underline{n}\}, b, \mu, \nu) Z^{S} .
$$

Then the global hard renormalization constant $Z^{H}$ is given as $Z^{H}=\left(Z^{D} Z^{S}\right)^{-1}$, and the non-global renormalization constant $\hat{Z}_{l m}$ is given as

$$
\hat{Z}_{l m}=Z_{l m}^{S} Z^{S}, \quad Z_{l m}^{H}=\hat{Z}_{l m} Z^{H} .
$$

Based on the above definitions, the RG equations of each ingredients are given as

$$
\begin{aligned}
& \frac{d}{d \ln \mu} \mathcal{H}_{m}(\{\underline{n}\}, Q, \mu)= \\
& \sum_{l=2}^{m} \mathcal{H}_{l}(\{\underline{n}\}, Q, \mu)\left\{\left[\Gamma_{\text {cusp }}\left(\alpha_{s}\right) \ln \frac{Q^{2}}{\mu^{2}}-2 \gamma^{D_{q}}\left(\alpha_{s}\right)-\gamma^{S}\left(\alpha_{s}\right)\right] \delta_{l m} \mathbf{1}-\hat{\boldsymbol{\Gamma}}_{l m}(\{\underline{n}\}, \mu)\right\} \\
& \frac{d}{d \ln \mu} \mathcal{S}_{l}(\{\underline{n}\}, b, \mu, \nu)= \\
& \sum_{m=l}^{\infty}\left\{\left[-\Gamma_{\text {cusp }}\left(\alpha_{s}\right) \ln \frac{\nu^{2}}{\mu^{2}}+\gamma^{S}\left(\alpha_{s}\right)\right] \delta_{l m} \mathbf{1}+\hat{\boldsymbol{\Gamma}}_{l m}(\{\underline{n}\}, \mu)\right\} \hat{\otimes} \mathcal{S}_{m}(\{\underline{n}\}, b, \mu, \nu) \\
& \frac{d}{d \ln \mu} D_{h / i}(z, b, \mu, \nu)=\left[\Gamma_{\text {cusp }}\left(\alpha_{s}\right) \ln \frac{\nu^{2}}{Q^{2}}+2 \gamma^{D_{q}}\left(\alpha_{s}\right)\right] D_{h / i}(z, b, \mu, \nu)
\end{aligned}
$$

where the anomalous dimensions are derived via

$$
\Gamma=-Z^{-1} \frac{d}{d \ln \mu} Z .
$$

Besides, both soft and TMD FF are suffering from the rapidity divergence, and the corresponding Rapidity-RG equations for them are

$$
\begin{aligned}
& \frac{d}{d \ln \nu} \mathcal{S}_{l}(\{\underline{n}\}, b, \mu, \nu)=\gamma_{\nu}^{S}\left(\alpha_{s}\right) \mathcal{S}_{l}(\{\underline{n}\}, b, \mu, \nu) . \\
& \frac{d}{d \ln \nu} D_{h / i}(z, b, \mu, \nu)=\gamma_{\nu}^{D}\left(\alpha_{s}\right) D_{h / i}(z, b, \mu, \nu) .
\end{aligned}
$$


Similarly, the rapidity anomalous dimension is defined as

$$
\gamma_{\nu}=-Z^{-1} \frac{d}{d \ln \nu} Z
$$

The expressions for the one-loop global anomalous dimensions have been given in the previous section. After solving the RG equations, we can obtain an all-order resummation formula. At the NLL accuracy, it has the form as

$$
\begin{aligned}
\frac{d \sigma}{d z_{h} d^{2} \overrightarrow{j_{T}}}= & \sigma_{0} \sum_{i=q, \bar{q}} e_{i}^{2} \int_{0}^{\infty} \frac{b d b}{2 \pi} J_{0}\left(b j_{T} / z_{h}\right) e^{-S_{\mathrm{pert}}\left(\mu_{b *}, \mu_{h}\right)-S_{\mathrm{NP}}\left(b, Q_{0}, Q\right)} \\
& \times \frac{1}{z_{h}^{2}} D_{h / i}\left(z_{h}, \mu_{b *}\right) U_{\mathrm{NG}}\left(\mu_{b *}, \mu_{h}\right) .
\end{aligned}
$$

In comparison with the resummed formalism in eq. (2.30), we have the non-global evolution function $U_{\mathrm{NG}}$, which is given as

$$
U_{\mathrm{NG}}\left(\mu_{b *}, \mu_{h}\right)=\frac{1}{N_{c}} \sum_{l=2}^{\infty} \operatorname{Tr}_{c}\left[\mathcal{H}_{l}\left(\left\{\underline{n}^{\prime}\right\}, Q, \mu_{h}\right) \otimes \sum_{m \geq l}^{\infty} \boldsymbol{U}_{l m}\left(\{\underline{n}\}, \mu_{h}, \mu_{b *}\right) \hat{\otimes} \mathcal{S}_{m}\left(\{\underline{n}\}, b, \mu_{b *}\right)\right],
$$

where $U_{\mathrm{NG}}$ is the evolution function for the non-global parts. At the LL accuracy and the large- $N_{c}$ limit, one can calculate it using the parton shower algorithms in $[51,73]$ or the numerical solution of the BMS equations [53]. For the convenience of our numerical calculations in the next section, however, we choose the parametrization given in [51]

$$
U_{\mathrm{NG}}\left(\mu_{b *}, \mu_{h}\right)=\exp \left[-C_{A} C_{F} \frac{\pi^{2}}{3} u^{2} \frac{1+(a u)^{2}}{1+(b u)^{c}}\right],
$$

with $a=0.85 C_{A}, b=0.86 C_{A}, c=1.33$, and

$$
u=\int_{\mu_{b} *}^{\mu_{h}} \frac{d \mu}{\mu} \frac{\alpha_{s}(\mu)}{2 \pi}=\frac{1}{\beta_{0}} \ln \left[\frac{\alpha_{s}\left(\mu_{b *}\right)}{\alpha_{s}\left(\mu_{h}\right)}\right],
$$

where $\beta_{0}=\frac{11}{3} C_{A}-\frac{4}{3} T_{F} n_{f}$, with $T_{F}=1 / 2$.

For the differential cross section in the threshold limit, we find that the same nonglobal evolution function $U_{\mathrm{NG}}$ arises. We thus write the resummed formalism at the NLL in the threshold limit $z_{h} \rightarrow 1$ as

$$
\begin{aligned}
\frac{d \sigma}{d z_{h} d^{2} \vec{j}_{T}}= & \sigma_{0} \sum_{i=q, \bar{q}} e_{i}^{2} \int_{0}^{\infty} \frac{b d b}{2 \pi} J_{0}\left(b j_{T} / z_{h}\right) \int_{z_{h}}^{1} \frac{d z}{z} e^{-\hat{S}_{\mathrm{pert}}\left(\mu_{b *}, \mu_{h}\right)-\hat{S}_{\mathrm{NP}}\left(b, Q_{0}, Q\right)} \\
& \times \frac{1}{z_{h}^{2}} \frac{e^{-2 \gamma_{E} \eta}}{\Gamma(2 \eta)} \frac{1}{1-z} D_{h / i}\left(z_{h} / z, \mu_{h}\right) U_{\mathrm{NG}}\left(\mu_{b *}, \mu_{h}\right)
\end{aligned}
$$

\section{Numerical results}

In this section, we will study the differential cross sections and Gaussian widths of the transverse momentum distribution for the single inclusive charged pion production (sum 

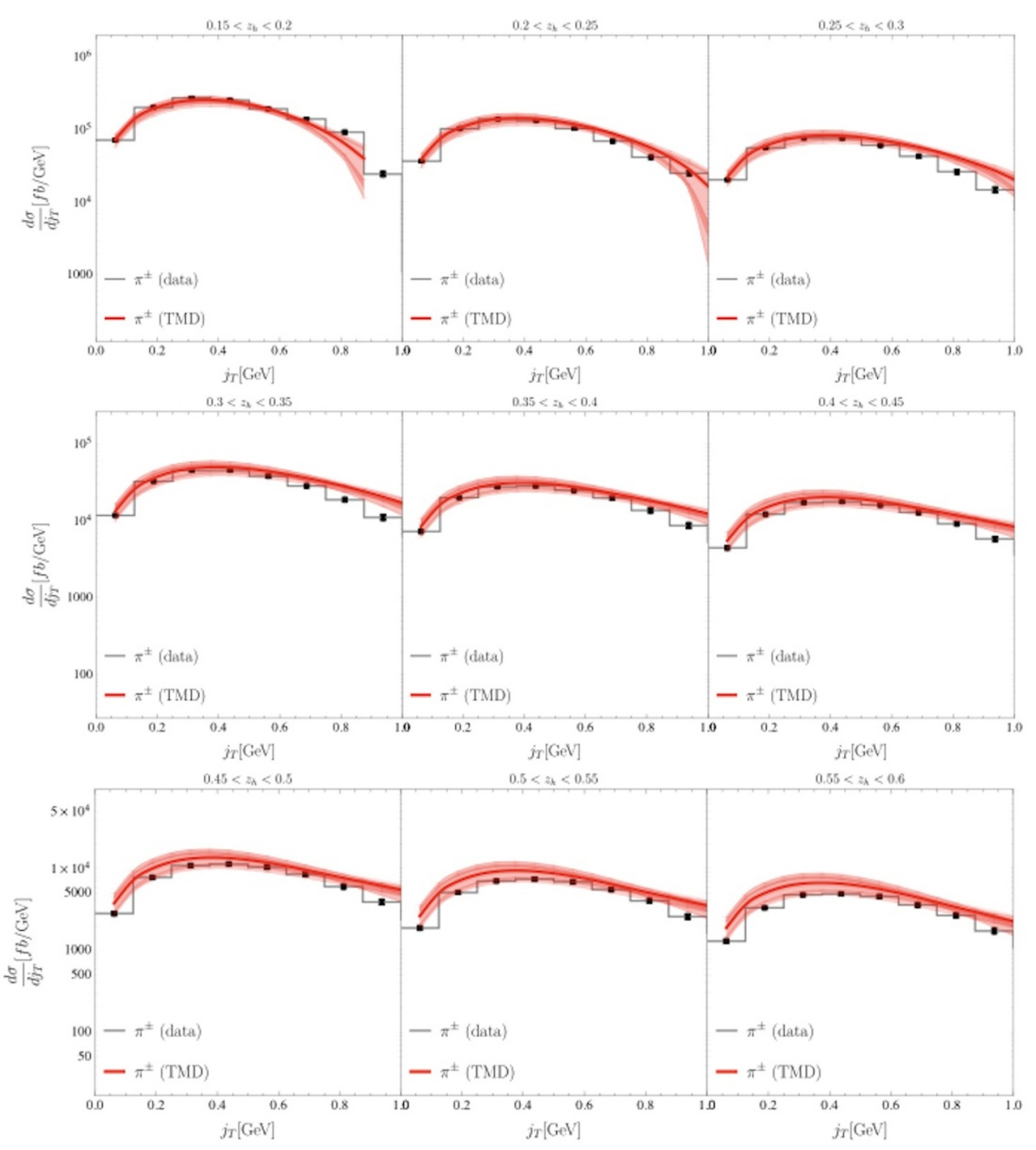

Figure 3. Differential cross sections for the charged pion as a function of $j_{T}$ and different $z_{h}$ bins with $\Delta z_{h}=0.05$ interval in the region $0.15<z_{h}<0.6$. In each plot, TMD resummation is applied and data points (black dots and histogram with error bars) from Belle collaboration [48] are shown for comparison. The red bands indicate theoretical uncertainties from the hard scale and $\mathrm{FF}$ variations as illustrated in the text.

of $\pi^{+}$and $\pi^{-}$) in electron-positron annihilation process, $e^{+} e^{-} \rightarrow \pi^{ \pm}+X$, based on the factorization and NLL resummation formula given in section 3. For parton-to-pion fragmentation functions, we use the 2014 DSS analysis [74], where the uncertainties were determined based on the standard iterative Hessian method. Note that Belle data [48] was originally presented in different thrust bins, in $0.5<T<0.7,0.7<T<0.8,0.8<T<0.9$, $0.9<T<0.95$ and $0.95<T<1.0$. Since the theoretical formalism we have developed in this paper is inclusive in the thrust variable, we thus combine the experimental data to obtain the results for the entire region $0.5<T<1.0$. The data shown in this section are all the ones obtained via such a combination procedure. The errors of the data sets are also combined weighted by corresponding thrust bins. 


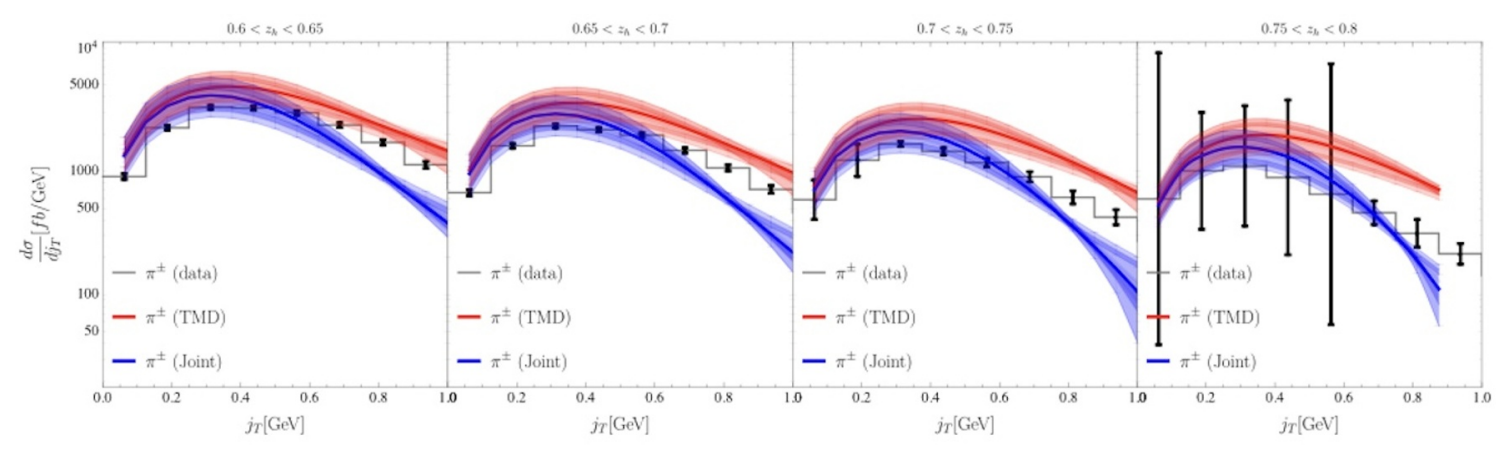

Figure 4. Transverse momentum $j_{T}$ distribution given by TMD resummation (red band) and the joint TMD and threshold resummation (blue band) for the charged pion production with $z_{h}$ bins $0.65<z_{h}<0.7,0.7<z_{h}<0.75,0.75<z_{h}<0.8$ and $0.8<z_{h}<0.85$ from left to right. Results are shown in comparison with Belle data [48] in each pad.

Figure 3 shows the differential cross sections for pion production in $e^{+} e^{-}$collision as a function of the pion transverse momentum $j_{T}$, in different $z_{h}$ bins at the center-of-mass energy $\sqrt{s}=10.58 \mathrm{GeV}$. To estimate the theoretical uncertainties, we vary the hard scale $\mu_{h}$ by a factor of two around its default value independently for each replica of partonto-pion FFs at 90\% confidence level determined in [74], and then the total uncertainties are given by the envelope of all the variations. One could also vary the scale $\mu_{b *}$, which would shift value of $b_{\max }$ and thus generate a larger uncertainty band. In particular the scale $\mu_{b *}$ is connected with non-perturbative Sudakov factor (2.29) that is only fitted at the canonical scale $\mu_{b *}=2 e^{-\gamma_{E}} / b_{*}$. We therefore do not include theoretical uncertainties from $\mu_{b *}$ variations. The hadron transverse momentum with respected to the thrust axis is given in $0<j_{T}<1.0 \mathrm{GeV}$ for each plot. The energy fraction region $0.1<z_{h}<0.65$ is divided into eleven sub-regions with $\Delta z_{h}=0.05$ for each panel. As seen clearly in the figure, for the intermediate $z_{h}$ region $\left(z_{h} \lesssim 0.5\right)$, the evaluations based on TMD resummation in eq. (3.15) are in good agreement with the data. ${ }^{4}$ On the other hand, as $z_{h}$ becomes relatively large $\left(z_{h} \gtrsim 0.5\right)$ and thus approaches the threshold limit, the agreement becomes worse, which indicates the potential importance of the threshold resummation effect.

In figure 4 we compare the differential cross sections obtained by using two resummations schemes: transverse momentum resummation (shown in red bands) and joint transverse momentum and threshold resummation (shown in blue bands), where the band sizes indicate theoretical uncertainties from the hard scale and FF variations as discussed in the figure 3 . The hadron transverse momentum with respect to the thrust axis is given in $0<j_{T}<1.0 \mathrm{GeV}$ region. The energy fraction regions are $0.65<z_{h}<0.7,0.7<z_{h}<0.75$, $0.75<z_{h}<0.8$ and $0.8<z_{h}<0.85$ from left to right. In figure $4, z_{h}$ bins are larger than those in figure 3 where the threshold logarithms are making some difference, thus compared to TMD resummation, we see that joint resummation has a better performance in these $z_{h}$ bins, especially in the small $j_{T}$ region. As $z_{h}$ gets larger, the consistency between joint

\footnotetext{
${ }^{4}$ We have included an overall normalization of 0.25 in our theory to match the experimental data. Such a normalization is consistent with what is fitted in [50], after taking into account a difference of $N_{c}$ factor in the overall normalization.
} 


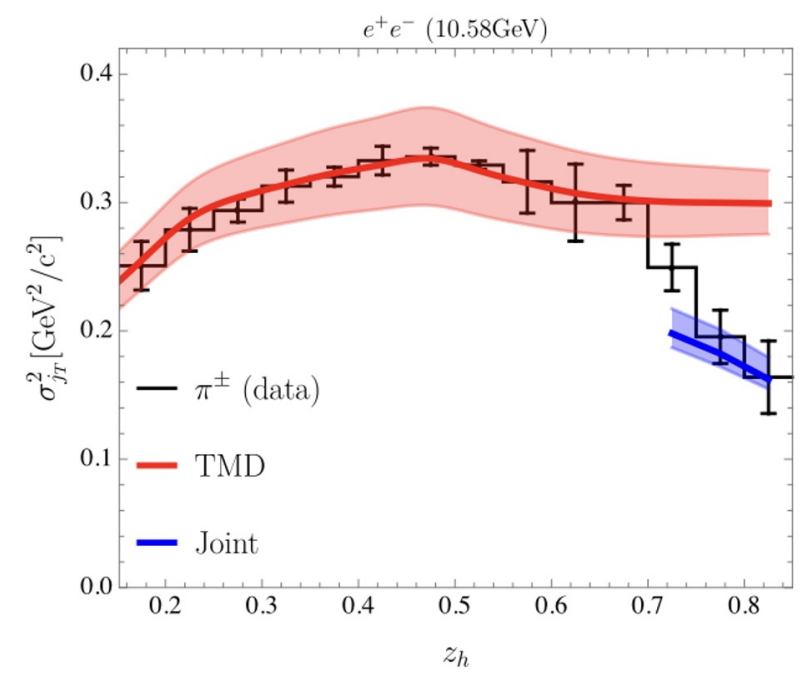

Figure 5. Gaussian widths for pion using TMD resummation (red band) and joint resummation (blue band) as a function of $z_{h}$ in the thrust bin $0.5<T<1.0$. Data points are constructed by charged pion differential cross sections measured at Belle detector.

resummation results and data gets better with decreasing Gaussian width. The jointly resummed differential cross section decreases faster, indicating a smaller Gaussian width value, which is more consistent with experimental data compared to the results with only transverse momentum resummed, where shapes are almost the same for the four $z_{h}$ bins in such a large $z_{h}$ region.

To see the change of $j_{T}$ width as a function of $z_{h}$, we fit the cross section $d \sigma / d z_{h} d^{2} \vec{j}_{T}$ as a function of $j_{T}^{2}$,

$$
\frac{d \sigma}{d z_{h} d^{2} \vec{j}_{T}} \propto \frac{1}{\pi \sigma_{j_{T}}^{2}} \exp \left(-j_{T}^{2} / \sigma_{j_{T}}^{2}\right)
$$

and reconstruct the Gaussian width $\sigma_{j_{T}}^{2}$ for both theory and experimental data. We compute Gaussian width as a function of fractional energy $z_{h}$ using both TMD resummation (red curve) and joint resummation (blue curve). In figure 5 , for small $z_{h}$ region $\left(z_{h}<0.5\right.$ ), the logarithmically enhanced contribution origins from $\ln \left(Q / j_{T}\right)$, thus transverse momentum resummed cross section $\sigma_{j_{T}}^{2}$ fits the data well. As the value of $z_{h}$ is increased, for the TMD factorization theorem in eq. (3.15), dependence on $z_{h}$ becomes weak, leading to a plateau at the tail region. On the other hand, for the factorization theorem with joint resummation in eq. (3.19), where transverse momentum and threshold logarithms are jointly resummed, the cross section sharply decreases as $z_{h}$ increases, indicating a better fit for this region. Generally speaking, for kinematic regions distinguished by $z_{h}$ bins, adopting TMD resummation in intermediate $z_{h}$ regions while making use of joint resummation for large $z_{h}$ bins can lead to excellent agreement with measurement for $e^{+} e^{-} \rightarrow \pi X$ process, suggesting our factorization and resummation formula results in a reasonable approach for describing single inclusive hadron production at the electron-positron colliders. 


\section{Conclusion}

Single inclusive hadron production at the $e^{+} e^{-}$colliders provide a new opportunity to study transverse momentum dependent fragmentation functions (TMD FFs), which are important to understand the 3D structure for the hadrons and the non-perturbative QCD. Belle collaboration has performed the first measurement for this observable, $e^{+} e^{-} \rightarrow h\left(z_{h}, j_{T}\right)+$ $X$, where $z_{h}$ is the energy fraction for the hadron, while the hadron's transverse momentum $j_{T}$ is measured with respect to the thrust axis determined by the hadronic event shape. We develop a TMD factorization formalism for such an observable, which resums logarithms $\ln \left(Q / j_{T}\right)$. Realizing the non-global nature of the observable, our factorization formalism involves a multi-Wilson line structure, which allows us to resum both global and nonglobal logarithms. Besides, as the increasing of the energy fraction $z_{h}$ of the hadron, the threshold soft gluon enhancement effects become more and more important, which require us to perform joint TMD $\left(\sim \ln \left(Q / j_{T}\right)\right)$ and threshold $\left(\sim \ln \left(1-z_{h}\right)\right)$ resummation. We apply the formalism proposed in $[68,69]$ based on SCET + framework [66] to obtain factorization and resummation formula in the joint limit.

In the end, we find that the TMD resummation formula gives a good description of the $j_{T}$ distribution as $z_{h}<0.65$. For large $z_{h}>0.65$ region, to describe the data we need to include threshold resummation effects. Especially, we find that the Gaussian width of the $j_{T}$ distribution given by the TMD formalism freeze to a certain value which is not consistent with the measurement. While after including joint threshold and TMD resummation effects, the theoretical predictions are consistent with the data very well.

In the present work, we obtained the perturbative resummed cross section at the next-to-leading logarithmic (NLL) accuracy. In future work, we will include higher-order resummation effects using method developed in [75]. Especially, in this case beyond the NLL level, the gluon TMD FF will also contribute to the cross section as shown in (3.3), it will be interesting to study its effects.

\section{Acknowledgments}

We thank Ralf Seidl for discussions on the combination of Belle data with different thrust bins and thank Daniele Anderle and Anselm Vossen for useful discussions. This work is supported by the National Science Foundation under Grant No. PHY-1720486 and CAREER award PHY-1945471 (Z.K., D.Y.S., and F.Z.), and by the Center for Frontiers in Nuclear Science of Stony Brook University and Brookhaven National Laboratory (D.Y.S.). This work is also supported within the framework of the TMD Topical collaboration.

Open Access. This article is distributed under the terms of the Creative Commons Attribution License (CC-BY 4.0), which permits any use, distribution and reproduction in any medium, provided the original author(s) and source are credited. 


\section{References}

[1] A. Accardi et al., Electron Ion Collider: The Next QCD Frontier: Understanding the glue that binds us all, Eur. Phys. J. A 52 (2016) 268 [arXiv:1212.1701] [INSPIRE].

[2] D. Boer et al., Gluons and the quark sea at high energies: Distributions, polarization, tomography, arXiv: 1108.1713 [INSPIRE].

[3] A. Prokudin, Y. Hatta, Y. Kovchegov and C. Marquet eds., Probing Nucleons and Nuclei in High Energy Collisions, proceedings of the INT Program INT-18-3, Seattle, WA, U.S.A., 1 October-16 November 2018, World Scientific (2020).

[4] X. Liu, F. Ringer, W. Vogelsang and F. Yuan, Lepton-jet Correlation in Deep Inelastic Scattering, Phys. Rev. D 102 (2020) 094022 [arXiv:2007.12866] [InSPIRE].

[5] Jefferson Lab Angular Momentum collaboration, Origin of single transverse-spin asymmetries in high-energy collisions, Phys. Rev. D 102 (2020) 054002 [arXiv:2002.08384] [INSPIRE].

[6] A. Bacchetta, F. Delcarro, C. Pisano and M. Radici, The three-dimensional distribution of quarks in momentum space, arXiv:2004.14278 [INSPIRE].

[7] A. Bacchetta et al., Transverse-momentum-dependent parton distributions up to $N^{3} L L$ from Drell-Yan data, JHEP 07 (2020) 117 [arXiv:1912.07550] [INSPIRE].

[8] I. Scimemi and A. Vladimirov, Non-perturbative structure of semi-inclusive deep-inelastic and Drell-Yan scattering at small transverse momentum, JHEP 06 (2020) 137 [arXiv: 1912.06532] [INSPIRE].

[9] X. Liu, F. Ringer, W. Vogelsang and F. Yuan, Lepton-jet Correlations in Deep Inelastic Scattering at the Electron-Ion Collider, Phys. Rev. Lett. 122 (2019) 192003 [arXiv: 1812.08077] [INSPIRE].

[10] Y.-T. Chien, R. Rahn, S. Schrijnder van Velzen, D.Y. Shao, W.J. Waalewijn and B. Wu, Azimuthal angle for boson-jet production in the back-to-back limit, arXiv:2005.12279 [INSPIRE].

[11] Y.-T. Chien, D.Y. Shao and B. Wu, Resummation of Boson-Jet Correlation at Hadron Colliders, JHEP 11 (2019) 025 [arXiv: 1905.01335] [INSPIRE].

[12] S. Fleming, Y. Makris and T. Mehen, An effective field theory approach to quarkonium at small transverse momentum, JHEP 04 (2020) 122 [arXiv:1910.03586] [INSPIRE].

[13] M.G.A. Buffing, Z.-B. Kang, K. Lee and X. Liu, A transverse momentum dependent framework for back-to-back photon + jet production, arXiv:1812.07549 [INSPIRE].

[14] M.G. Echevarria, A. Idilbi, Z.-B. Kang and I. Vitev, QCD Evolution of the Sivers Asymmetry, Phys. Rev. D 89 (2014) 074013 [arXiv: 1401.5078] [INSPIRE].

[15] D. Boer, C. Lorcé, C. Pisano and J. Zhou, The gluon Sivers distribution: status and future prospects, Adv. High Energy Phys. 2015 (2015) 371396 [arXiv:1504.04332] [INSPIRE].

[16] A. Bacchetta, M. Diehl, K. Goeke, A. Metz, P.J. Mulders and M. Schlegel, Semi-inclusive deep inelastic scattering at small transverse momentum, JHEP 02 (2007) 093 [hep-ph/0611265] [INSPIRE].

[17] Z.-B. Kang, A. Prokudin, P. Sun and F. Yuan, Extraction of Quark Transversity Distribution and Collins Fragmentation Functions with QCD Evolution, Phys. Rev. D 93 (2016) 014009 [arXiv: 1505.05589] [INSPIRE]. 
[18] M.G. Echevarria, Y. Makris and I. Scimemi, Quarkonium TMD fragmentation functions in NRQCD, JHEP 10 (2020) 164 [arXiv:2007.05547] [INSPIRE].

[19] D. Callos, Z.-B. Kang and J. Terry, Extracting the transverse momentum dependent polarizing fragmentation functions, Phys. Rev. D 102 (2020) 096007 [arXiv:2003.04828] [INSPIRE].

[20] M. Arratia, Z.-B. Kang, A. Prokudin and F. Ringer, Jet-based measurements of Sivers and Collins asymmetries at the future electron-ion collider, Phys. Rev. D 102 (2020) 074015 [arXiv: 2007.07281] [INSPIRE].

[21] D. Neill, I. Scimemi and W.J. Waalewijn, Jet axes and universal transverse-momentumdependent fragmentation, JHEP 04 (2017) 020 [arXiv:1612.04817] [INSPIRE].

[22] Z.-B. Kang, A. Prokudin, F. Ringer and F. Yuan, Collins azimuthal asymmetries of hadron production inside jets, Phys. Lett. B $\mathbf{7 7 4}$ (2017) 635 [arXiv:1707.00913] [INSPIRE].

[23] Z.-B. Kang, X. Liu, F. Ringer and H. Xing, The transverse momentum distribution of hadrons within jets, JHEP 11 (2017) 068 [arXiv:1705. 08443] [INSPIRE].

[24] Z.-B. Kang, K. Lee, J. Terry and H. Xing, Jet fragmentation functions for Z-tagged jets, Phys. Lett. B 798 (2019) 134978 [arXiv:1906.07187] [INSPIRE].

[25] Z.-B. Kang, K. Lee and F. Zhao, Polarized jet fragmentation functions, Phys. Lett. B 809 (2020) 135756 [arXiv: 2005. 02398] [INSPIRE].

[26] D. Gutierrez-Reyes, Y. Makris, V. Vaidya, I. Scimemi and L. Zoppi, Probing TransverseMomentum Distributions With Groomed Jets, JHEP 08 (2019) 161 [arXiv:1907.05896] [INSPIRE].

[27] A. Metz and A. Vossen, Parton Fragmentation Functions, Prog. Part. Nucl. Phys. 91 (2016) 136 [arXiv: 1607.02521] [INSPIRE].

[28] J.C. Collins, D.E. Soper and G.F. Sterman, Transverse Momentum Distribution in Drell-Yan Pair and $W$ and $Z$ Boson Production, Nucl. Phys. B 250 (1985) 199 [INSPIRE].

[29] J. Collins, Foundations of perturbative QCD, in Cambridge Monographs on Particle Physics, Nuclear Physics and Cosmology 32, Cambridge University Press, Cambridge U.K. (2011) [INSPIRE].

[30] X.-d. Ji, J.-p. Ma and F. Yuan, QCD factorization for semi-inclusive deep-inelastic scattering at low transverse momentum, Phys. Rev. D 71 (2005) 034005 [hep-ph/0404183] [INSPIRE].

[31] J.C. Collins, Fragmentation of transversely polarized quarks probed in transverse momentum distributions, Nucl. Phys. B 396 (1993) 161 [hep-ph/9208213] [INSPIRE].

[32] D. Boer, P.J. Mulders and F. Pijlman, Universality of $T$ odd effects in single spin and azimuthal asymmetries, Nucl. Phys. B 667 (2003) 201 [hep-ph/0303034] [INSPIRE].

[33] J.C. Collins and D.E. Soper, Back-To-Back Jets in QCD, Nucl. Phys. B 193 (1981) 381 [Erratum ibid. 213 (1983) 545] [INSPIRE].

[34] D. Boer, R. Jakob and P.J. Mulders, Asymmetries in polarized hadron production in $e^{+} e^{-}$ annihilation up to order $1 / Q$, Nucl. Phys. B 504 (1997) 345 [hep-ph/9702281] [INSPIRE].

[35] D. Pitonyak, M. Schlegel and A. Metz, Polarized hadron pair production from electronpositron annihilation, Phys. Rev. D 89 (2014) 054032 [arXiv:1310.6240] [INSPIRE].

[36] P. Sun, J. Isaacson, C.P. Yuan and F. Yuan, Nonperturbative functions for SIDIS and Drell-Yan processes, Int. J. Mod. Phys. A 33 (2018) 1841006 [arXiv:1406.3073] [INSPIRE]. 
[37] M. Boglione, J. Collins, L. Gamberg, J.O. Gonzalez-Hernandez, T.C. Rogers and N. Sato, Kinematics of Current Region Fragmentation in Semi-Inclusive Deeply Inelastic Scattering, Phys. Lett. B 766 (2017) 245 [arXiv:1611.10329] [inSPIRE].

[38] F. Hautmann, I. Scimemi and A. Vladimirov, Non-perturbative contributions to vector-boson transverse momentum spectra in hadronic collisions, Phys. Lett. B 806 (2020) 135478 [arXiv: 2002.12810] [INSPIRE].

[39] J.C. Collins and A. Metz, Universality of soft and collinear factors in hard-scattering factorization, Phys. Rev. Lett. 93 (2004) 252001 [hep-ph/0408249] [INSPIRE].

[40] A. Bacchetta, F. Delcarro, C. Pisano, M. Radici and A. Signori, Extraction of partonic transverse momentum distributions from semi-inclusive deep-inelastic scattering, Drell-Yan and Z-boson production, JHEP 06 (2017) 081 [Erratum JHEP 06 (2019) 051] [arXiv: 1703.10157] [INSPIRE].

[41] C.W. Bauer, S. Fleming, D. Pirjol and I.W. Stewart, An Effective field theory for collinear and soft gluons: Heavy to light decays, Phys. Rev. D 63 (2001) 114020 [hep-ph/0011336] [INSPIRE].

[42] C.W. Bauer, D. Pirjol and I.W. Stewart, Soft collinear factorization in effective field theory, Phys. Rev. D 65 (2002) 054022 [hep-ph/0109045] [INSPIRE].

[43] C.W. Bauer, S. Fleming, D. Pirjol, I.Z. Rothstein and I.W. Stewart, Hard scattering factorization from effective field theory, Phys. Rev. D 66 (2002) 014017 [hep-ph/0202088] [INSPIRE].

[44] M. Beneke, A.P. Chapovsky, M. Diehl and T. Feldmann, Soft collinear effective theory and heavy to light currents beyond leading power, Nucl. Phys. B 643 (2002) 431 [hep-ph/0206152] [INSPIRE].

[45] T. Becher and M. Neubert, Drell-Yan Production at Small $q_{T}$, Transverse Parton Distributions and the Collinear Anomaly, Eur. Phys. J. C 71 (2011) 1665 [arXiv: 1007.4005] [INSPIRE].

[46] J.-y. Chiu, A. Jain, D. Neill and I.Z. Rothstein, The Rapidity Renormalization Group, Phys. Rev. Lett. 108 (2012) 151601 [arXiv:1104.0881] [INSPIRE].

[47] M.G. Echevarria, A. Idilbi and I. Scimemi, Factorization Theorem For Drell-Yan At Low $q_{T}$ And Transverse Momentum Distributions On-The-Light-Cone, JHEP 07 (2012) 002 [arXiv: 1111.4996] [INSPIRE].

[48] BeLLE collaboration, Transverse momentum dependent production cross sections of charged pions, kaons and protons produced in inclusive $e^{+} e^{-}$annihilation at $\sqrt{s}=10.58 \mathrm{GeV}$, Phys. Rev. D 99 (2019) 112006 [arXiv: 1902.01552] [INSPIRE].

[49] M. Boglione, J.O. Gonzalez-Hernandez and R. Taghavi, Transverse parton momenta in single inclusive hadron production in $e^{+} e^{-}$annihilation processes, Phys. Lett. B 772 (2017) 78 [arXiv: 1704.08882] [INSPIRE].

[50] M. Soleymaninia and H. Khanpour, Transverse momentum dependent of charged pion, kaon, and proton/antiproton fragmentation functions from $e^{+} e^{-}$annihilation process, Phys. Rev. D 100 (2019) 094033 [arXiv:1907.12294] [INSPIRE].

[51] M. Dasgupta and G.P. Salam, Resummation of nonglobal QCD observables, Phys. Lett. B 512 (2001) 323 [hep-ph/0104277] [INSPIRE]. 
[52] G.F. Sterman, Resummations, power corrections and interjet radiation, Acta Phys. Polon. B 36 (2005) 389 [hep-ph/0410014] [INSPIRE].

[53] A. Banfi, G. Marchesini and G. Smye, Away from jet energy flow, JHEP 08 (2002) 006 [hep-ph/0206076] [INSPIRE].

[54] T. Becher, R. Rahn and D.Y. Shao, Non-global and rapidity logarithms in narrow jet broadening, JHEP 10 (2017) 030 [arXiv:1708.04516] [INSPIRE].

[55] S. Brandt, C. Peyrou, R. Sosnowski and A. Wroblewski, The Principal axis of jets. An Attempt to analyze high-energy collisions as two-body processes, Phys. Lett. 12 (1964) 57 [INSPIRE].

[56] A. Jain, M. Procura and W.J. Waalewijn, Fully-Unintegrated Parton Distribution and Fragmentation Functions at Perturbative $k_{T}$, JHEP 04 (2012) 132 [arXiv:1110.0839] [INSPIRE].

[57] M. Dasgupta and G.P. Salam, Accounting for coherence in interjet $E_{t}$ flow: A Case study, JHEP 03 (2002) 017 [hep-ph/0203009] [INSPIRE].

[58] T. Becher, B.D. Pecjak and D.Y. Shao, Factorization for the light-jet mass and hemisphere soft function, JHEP 12 (2016) 018 [arXiv: 1610.01608] [INSPIRE].

[59] T. Becher, M. Neubert, L. Rothen and D.Y. Shao, Factorization and Resummation for Jet Processes, JHEP 11 (2016) 019 [Erratum JHEP 05 (2017) 154] [arXiv:1605. 02737] [INSPIRE].

[60] E. Moffat, T.C. Rogers, N. Sato and A. Signori, Collinear factorization in wide-angle hadron pair production in $e^{+} e^{-}$annihilation, Phys. Rev. D 100 (2019) 094014 [arXiv:1909.02951] [INSPIRE].

[61] J.-Y. Chiu, A. Jain, D. Neill and I.Z. Rothstein, A Formalism for the Systematic Treatment of Rapidity Logarithms in Quantum Field Theory, JHEP 05 (2012) 084 [arXiv:1202.0814] [INSPIRE].

[62] M.A. Ebert, I.W. Stewart and Y. Zhao, Towards Quasi-Transverse Momentum Dependent PDFs Computable on the Lattice, JHEP 09 (2019) 037 [arXiv:1901.03685] [INSPIRE].

[63] M.G. Echevarria, I. Scimemi and A. Vladimirov, Unpolarized Transverse Momentum Dependent Parton Distribution and Fragmentation Functions at next-to-next-to-leading order, JHEP 09 (2016) 004 [arXiv: 1604.07869] [INSPIRE].

[64] M.-X. Luo, X. Wang, X. Xu, L.L. Yang, T.-Z. Yang and H.X. Zhu, Transverse Parton Distribution and Fragmentation Functions at NNLO: the Quark Case, JHEP 10 (2019) 083 [arXiv: 1908.03831] [INSPIRE].

[65] Z.-B. Kang, A. Prokudin, N. Sato and J. Terry, Efficient Fourier Transforms for Transverse Momentum Dependent Distributions, Comput. Phys. Commun. 258 (2021) 107611 [arXiv: 1906.05949] [INSPIRE].

[66] C.W. Bauer, F.J. Tackmann, J.R. Walsh and S. Zuberi, Factorization and Resummation for Dijet Invariant Mass Spectra, Phys. Rev. D 85 (2012) 074006 [arXiv:1106.6047] [InSPIRE].

[67] M. Procura, W.J. Waalewijn and L. Zeune, Resummation of Double-Differential Cross Sections and Fully-Unintegrated Parton Distribution Functions, JHEP 02 (2015) 117 [arXiv:1410.6483] [INSPIRE]. 
[68] Y. Li, D. Neill and H.X. Zhu, An exponential regulator for rapidity divergences, Nucl. Phys. $B 960$ (2020) 115193 [arXiv: 1604.00392] [INSPIRE].

[69] G. Lustermans, W.J. Waalewijn and L. Zeune, Joint transverse momentum and threshold resummation beyond NLL, Phys. Lett. B 762 (2016) 447 [arXiv:1605.02740] [INSPIRE].

[70] G. Sterman and M. Zeng, Quantifying Comparisons of Threshold Resummations, JHEP 05 (2014) 132 [arXiv: 1312.5397] [INSPIRE].

[71] T. Becher and M. Neubert, Threshold resummation in momentum space from effective field theory, Phys. Rev. Lett. 97 (2006) 082001 [hep-ph/0605050] [INSPIRE].

[72] M. Boglione and A. Simonelli, Universality-breaking effects in $e^{+} e^{-}$hadronic production processes, arXiv:2007.13674 [INSPIRE].

[73] M. Balsiger, T. Becher and D.Y. Shao, Non-global logarithms in jet and isolation cone cross sections, JHEP 08 (2018) 104 [arXiv: 1803.07045] [INSPIRE].

[74] D. de Florian, R. Sassot, M. Epele, R.J. Hernández-Pinto and M. Stratmann, Parton-to-Pion Fragmentation Reloaded, Phys. Rev. D 91 (2015) 014035 [arXiv:1410.6027] [INSPIRE].

[75] M. Balsiger, T. Becher and D.Y. Shao, NLL' resummation of jet mass, JHEP 04 (2019) 020 [arXiv: 1901.09038] [INSPIRE]. 